\title{
The Evaluation of the Use of a Paper Tracking Form to Improve Provider Adherence to Lipid Guidelines
}

Diane Ladd

West Virginia University

Follow this and additional works at: https://researchrepository.wvu.edu/etd

\section{Recommended Citation}

Ladd, Diane, "The Evaluation of the Use of a Paper Tracking Form to Improve Provider Adherence to Lipid Guidelines" (2011). Graduate Theses, Dissertations, and Problem Reports. 3073.

https://researchrepository.wvu.edu/etd/3073

This Dissertation is protected by copyright and/or related rights. It has been brought to you by the The Research Repository @ WVU with permission from the rights-holder(s). You are free to use this Dissertation in any way that is permitted by the copyright and related rights legislation that applies to your use. For other uses you must obtain permission from the rights-holder(s) directly, unless additional rights are indicated by a Creative Commons license in the record and/ or on the work itself. This Dissertation has been accepted for inclusion in WVU Graduate Theses, Dissertations, and Problem Reports collection by an authorized administrator of The Research Repository @ WVU.

For more information, please contact researchrepository@mail.wvu.edu. 
The Evaluation of the Use of a Paper Tracking Form to Improve Provider Adherence to Lipid Guidelines

Diane Ladd, MSN, FNP-BC

\author{
Doctoral Research Project submitted to the \\ School of Nursing \\ at West Virginia University \\ in partial fulfillment of the requirements \\ for the degree of
}

Doctor of Nursing Practice

\author{
Susan McCrone, PhD, Chair \\ Lisa Hardman, $\mathrm{PhD}$ \\ Richard Ansinelli, MD
}

Morgantown, WV

2011

Keywords: provider, adherence, lipid, guidelines 


\begin{abstract}
The Evaluation of the Use of a Paper Tracking Form to Improve Provider Adherence to Lipid Guidelines
\end{abstract}

Diane Ladd, MSN, FNP-BC

Heart disease is the leading cause of death in men and women in the United States. Significant research into the risk factors for cardiovascular disease has been done, and the effect of hyperlipidemia on cardiovascular risk has been well documented. Recent research has shown that there is a lack of adherence to lipid guidelines by primary care providers. Failure to follow clinical guidelines for lipid disorders leads to increased cardiovascular morbidity and mortality. The objective of the project was to evaluate whether a simple, paper-based, lipid tracking form could improve provider adherence to current lipid guidelines. This descriptive study used a prepost test design. Baseline adherence to lipid guidelines was determined by chart review of a random sample of current practice patients. The lipid-tracking form was placed on the charts of all patients seen for routine office visits during a specified time frame. After the lipid-tracking form collection period was completed, a random sample of those patients was reviewed to reassess adherence to lipid guidelines. The post-implementation date was compared to the baseline data. The use of the lipid-tracking form did demonstrate an improvement in adherence to lipid guidelines as demonstrated by an increase in the number of patients who had a documented LDL goal $(\mathrm{p}=0.000)$. In practices without electronic medical records, this simple, inexpensive, paper-based tool can help providers improve adherence to lipid guidelines. 


\section{Acknowledgments}

In order to complete a task of this magnitude you need the support of many people and I would like to take this opportunity to thank those who supported, helped and encouraged me throughout this process.

My committee members: Dr. Susan McCrone, Dr. Lisa Harman and Dr. Richard Ansinelli who were a constant source of support and encouragement.

My classmates who know from experience what this process is like.

My family who always support my educational endeavors however many they may be.

My husband: Richard Engelman for always being there when I needed him and his support and confidence in me. 
Table of Contents

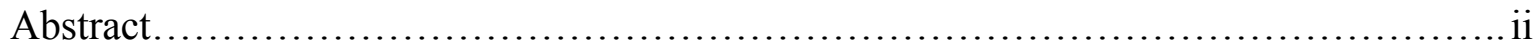

Acknowledgements.......................................................... iii

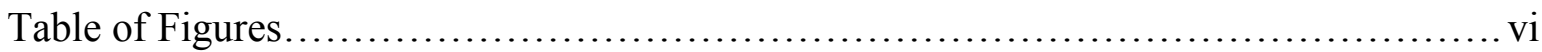

Table of Appendices........................................................ vii

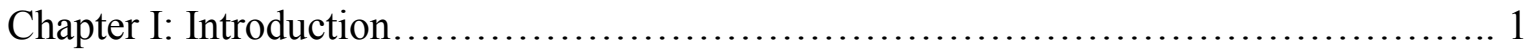

Background and Significance.......................................... 1

Statement of the problem $\ldots \ldots \ldots \ldots \ldots \ldots \ldots \ldots \ldots \ldots \ldots \ldots \ldots \ldots \ldots \ldots \ldots$

Description of the problem..................................... 1

Theoretical Framework...............................................4

Chapter II: Review of the Literature........................................... 10

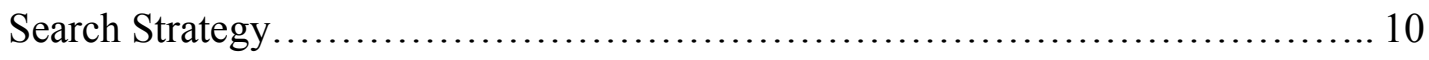

Inclusion Criteria...................................................... 10

Literature Review: Lipid Guideline Adherence............................ 10

Synthesis......................................................... 16

Congruence with Organizational Strategic Plan.............................. 18

Project Objectives.................................................... 18

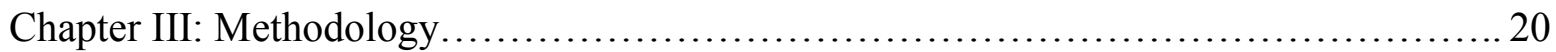

Project Design..................................................... 20

Resources............................................................ 20

Evidence of Key Site Support ......................................... 22

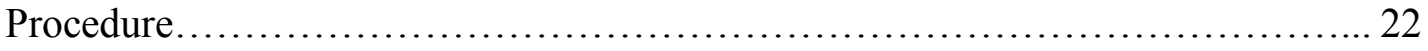

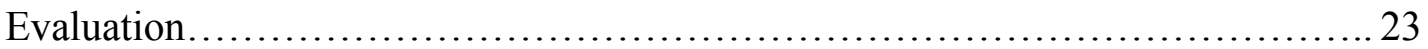




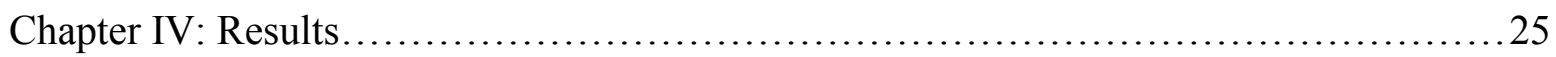

Samples Characteristics................................................. 25

Assessment of Baseline Adherence.......................................... 25

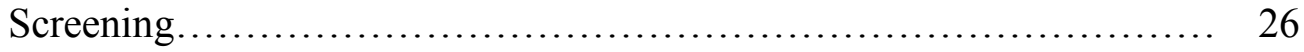

Assessment of the diagnosis of hyperlipidemia....................... 26

Documentation of LDL goal.................................... 27

Assessment of medication usage to treat hyperlipidemia............... 27

Follow-up.................................................... 28

Evaluation of Post Practice Change Adherence............................... 29

Documentation of LDL goal...................................... 29

Follow-up for hyperlipidemia................................... 30

Other analyses...................................................... $\quad 30$

Chapter V: Summary, Discussion \& Implications............................... 32

Congruence with the Theoretical Framework................................... 39

Adopter categories.............................................. 32

Factors that affect the rate of adoption.............................. 33

Diffusion of Innovations: Stages................................. 34

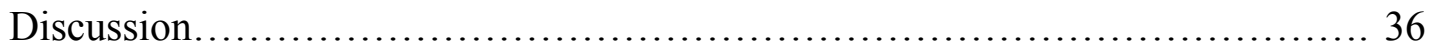

Limitations of the project........................................ 37

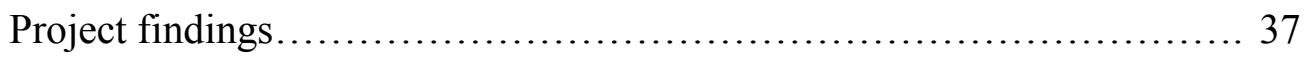

Implications............................................................. 38

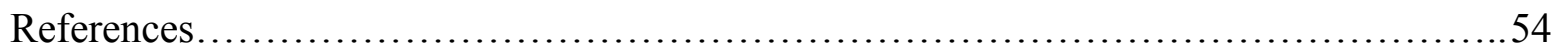




\section{List of Figures}

Figure 1: Average annual age-adjusted rate (deaths per 100,000) for people ages 35 years and older

Figure 2: Prevalence of elevated cholesterol and indices of increased cardiovascular risk broken down by gender and race

Figure 3: Rogers' Diffusion of Innovation

Figure 4: Ladd's adaption of Rogers' Diffusion of Innovation for use in implementing practice change.

Figure 5: Ladd's further adaptation of Rogers Diffusion of Innovations Theory for practice change

Figure 6: Diffusion of Innovation s-curve and normal distribution of adopter categories 


\section{List of Appendices}

Appendix A: Instructions for the Lipid Tracking Form Appendix B: Data Collection Form

Appendix C: Lipid Tracking Form

Appendix D: ATP-III Quick Reference Guide

Appendix E: Letter of Support

Appendix F: Framingham Risk Scores 


\section{Chapter I: Introduction}

Heart disease is the leading cause of death for men and women in the United States (Center for Disease Control and Prevention [CDC], 2009). Coronary heart disease (CHD), the narrowing of the coronary arteries due to fatty build-up of plaque, caused 425,425 deaths in the U.S. in 2006 (American Heart association [AHA], 2006). The American Heart Association and National Heart, Lung, and Blood Institute (NHLBI) estimated that the direct and indirect cost of cardiovascular disease in the U.S. for 2009 was 475.3 billion dollars (AHA, 2010). Direct costs include healthcare providers and medications, and indirect costs refer to the loss of productivity from disability and death. The economic cost can be measured, but the true human cost of debility, suffering, and death is incalculable.

\section{Background and Significance}

\section{Statement of the problem.}

Failure to follow clinical guidelines for hyperlipidemia (HLD) screening, treatment, and follow-up leads to increased cardiovascular morbidity and mortality, which is a significant healthcare burden in money, time, and disability.

\section{Description of the problem.}

Heart disease is the leading cause of death in most ethnic groups in the United States including African Americans, American Indians or Alaska Natives, Hispanics, and Caucasians, while for Asians, heart disease is second to cancer (CDC, 2009). The heart disease death rates per 100,000 persons in the United States are highest in Blacks (662) and second highest in Caucasians (529) (CDC, 2009). The heart disease death rates in Michigan (per 100,000), where this project occurred, are highest in ethnic populations. American Indians had the highest heart 
disease death rate (805), and African Americans had the second highest heart disease death rate (709), while whites had a rate of 561. Midland County Michigan's heart disease death rate was highest in American Indians (759), second highest in African Americans (659), and third highest in whites (580). Overall Midland's heart disease death rate was higher than the United States for total population (585 v. 536), more than double for American Indian (759 v. 352), more or less equivalent for African Americans (659 v. 662), higher for Hispanics (409 v. 348) and higher for whites (580 v. 529) (Figure 1). Sager et al. (2010) calculated that adherence to lipid guidelines for cholesterol targets would prevent 50-80 heart attacks, strokes, and cardiovascular disease deaths per 1000 persons over a ten-year period.

The INTERHEART study (Yusuf et al., 2004), a large multi-ethnic case-control study, that was conducted in 52 countries has shown that there are nine easily measured cardiovascular risk factors that account for over $90 \%$ of the risk of acute myocardial infarction and other cardiovascular problems (lipids, smoking, hypertension, diabetes, obesity, diet, physical activity, alcohol consumption, and psychosocial factors). The most significant risk factor is hyperlipidemia (Yusuf et al., 2004). Hyperlipidemia is the presence of elevated lipid and lipoprotein levels in the blood. The link between lipid abnormalities especially increased lowdensity lipoprotein (LDL) levels and cardiovascular risk has been well established (Karthikeyan et al., 2009). LDL is the primary atherogenic lipid particle, and it is directly related to the development of atherosclerosis (Jones, 2010).

Data from the American Heart Association (2010) shows that approximately 102.2 million adults in the United States have total cholesterol levels over $200 \mathrm{mg} / \mathrm{dl}$. The prevalence of elevated total cholesterol $(>200 \mathrm{mg} / \mathrm{dl})$ is highest in Hispanics at $51.1 \%$. It is second highest in whites $45.0 \%$. Of the ethnicities studied, the prevalence of elevated total cholesterol $(>200$ 
$\mathrm{mg} / \mathrm{dl}$ ) was lowest in Blacks at 40.2\%. From those about 35.7 million have total cholesterol levels greater than $240 \mathrm{mg} / \mathrm{dl}$. Utilizing the National Health and Nutrition Examinations Survey (NHANES) data, the AHA further subdivided the data by ethnicity (Black, Hispanic, and White), gender, and measures of increased cardiovascular (CV) risk (total cholesterol $>240 \mathrm{mg} / \mathrm{dl}$, LDL $>130 \mathrm{mg} / \mathrm{dl}$, and HDL $<40 \mathrm{mg} / \mathrm{dl})$. Additional data for total cholesterol $>240 \mathrm{mg} / \mathrm{dl}$ showed the prevalence to be highest in Hispanics at $16.8 \%$, followed by Whites at $15.3 \%$, and then Blacks at $10.9 \%$. The prevalence of LDL $>130$ was found to be highest in Hispanics at $42.7 \%$, second highest in Blacks 34.4\% and lowest in Whites 31.5\%. In addition, the AHA also provided prevalence data for HDL $<40$. The highest prevalence was highest in Hispanics at 29.3\%, second in Whites at 25.4\%, and lowest in Blacks at 14.7\% (Figure 2).

Coronary heart disease is usually caused by atherosclerosis (a buildup of fatty material and plaque inside the coronary arteries). Atherosclerosis is progressive and begins early in life. Forensic data from the Pathologic Determinants of Atherosclerosis in Youth (PDAY) study has shown the presence of fatty streaks in segments of the arterial system of all teenagers autopsied (McGill \& McMahan, 1998). In addition, they found fatty plaques in the aorta and coronary arteries that increased with age. Despite decades of research on lipids and coronary risk, there is still controversy over which lipid measurement is the most accurate predictor of cardiovascular risk. Current guidelines from the National Cholesterol Education Program: Adult Treatment Panel-III (ATP-III) has identified low-density lipoprotein (LDL) as the primary target for lipidlowering therapy for the reduction cardiovascular risk (Pasternak, 2002).

There is sufficient evidence from clinical trials that identifies an association between a decrease in cardiovascular morbidity and mortality with the decrease in atherogenic particles (Jones, 2010). This has been demonstrated in patients with and without pre-existing 
cardiovascular disease (Corrao, Conti, Merlino, Catapano, \& Mancia, 2010). Despite the abundance of evidence demonstrating the benefits of lipid control, there is still a significant failure of providers to appropriately screen, treat, or assign appropriate goals for lipid management (Sager et al., 2010). Therefore, the purpose of this project is to evaluate the use of a paper-based lipid tracking tool on provider adherence to lipid lowering guidelines.

\section{Theoretical Framework}

One theory that can be used to guide the implementation of new guidelines, protocols, or practice changes is Rogers' Diffusion of Innovations Theory (Rogers, 1962). This theory is used to describe how, why, and at what rate innovations are adopted and disseminated. Contributions from other disciplines impacting this theory include: anthropology, sociology, education, and industrial and medical sociology (Ryan, 1943). One of basic premises of this theory is that innovation is adopted slowly during the beginning of the process then, once it is perceived to be beneficial, it is adopted more quickly. There are distinct elements in the Diffusion of Innovations Theory. They include: types of communication, time, rate of adoption, and the type of system or group for which the innovation is intended. For this capstone project, the site specific needs and deficiencies were taken into consideration. Communication regarding the project was in written form, as well as verbal, to provide concrete information and references for the staff. The type of system (small private practice) was taken into consideration in the project design.

Rogers' Diffusion of Innovations Theory is a five-step process. The five steps are: (1)

knowledge; (2) persuasion; (3) decision; (4) implementation; and 5) confirmation (Figure 3). The knowledge step involves gaining and recalling knowledge about the innovation. The persuasion step is when an individual becomes interested in the innovation, shares with others the positive role of the innovation within the group, and gathers support for the innovation within 
the system. During the decision making step, additional information is gathered and the innovation is initially adopted or rejected. The implementation step is when the innovation is utilized on a routine basis, and its usefulness and barriers can be determined. The fifth step of confirmation is when the group recognizes the benefits and integrates the innovation into the systems ongoing routine (Sharma \& Kanekar, 2008; Martin, 2009). This investigator has identified other steps that may be necessary when implementing guidelines, protocols, or practice changes. Rogers (1962) proposed that in the decision step the innovation will either be adopted or rejected. This investigator proposed that rather than outright rejection, the next step, if the innovation is not adopted, would be initial rejection. After initial rejection, there should be a reevaluation of the proposed innovation to assess its lack of adoptability. If it is determined that the innovation is still appropriate to the system, and should be adopted, then it should be reintroduced to the system beginning at the knowledge step. However, if it is determined that the innovation is not appropriate for the system, the last step would be final rejection. Once an innovation has gotten to the final rejection step, it should not be reintroduced back into the same system without changes (Figure 4).

Another significant factor in the use of this theory is identifying the rate of adoption. Each innovation is adopted at a different rate, and the speed at which the new innovation is adopted is determined, in part, by the adopter categories. Rogers (1962) describes five adopter categories: innovators, early adopters, early majority, late majority, and laggards. Innovators are usually the first to adopt an intervention. They are usually risk takers and tend to be younger in age. Early adopters are the second fastest category of adopters. They tend to have a high degree of leadership. The early majority are individuals who adopt after varying lengths of exposure to the innovation. These adopters take longer than the first two groups and tend to be slower in 
adopting. The late majority adopts the innovation only after other members of the group have adopted it. The late majority tend to be skeptical and wait to see what others have done. The laggards are the last to adopt an innovation and are generally averse to change. The breakdown of these categories forms a normal distribution curve (Figure 5).

In addition to the adopter categories, Rogers (1962) has described factors that influence the rate of adoption. These include: relative advantage, compatibility, complexity, trialability/testability, and observability. The relative advantage is the perceived advantage of the innovation within the group or system. Compatibility refers to the amount of compatibility the proposed innovation has within the already existing system or structure as well as values and needs of the adopters. Complexity involves not only the amount of work required to incorporate innovation within the group or system but also the perceived barriers to adopting the innovation. Trialability/testability refers to the amount to which an innovation can be tried on a limited basis. Observability is the amount to which the results of the innovation are visible to others. It is easier for people to adopt and accept an innovation if they can easily see the results (Decivita \& Dasgupta, 2007). The lipid-tracking tool provides an at-a-glance record that shows the progress (or lack thereof) of the patient lipid control and increases the observability of the innovation (practice change). In addition, the at-a-glance style of the form will reinforce the relative advantage (not having to look through the chart for history and lipid trends) of the innovation. The innovation is not complex, and there are no significant barriers such as need for specialized knowledge or technology.

This theory was useful in implementing structured screening, treatment, and follow-up protocols and practice changes for the evaluation and treatment of hyperlipidemia. The first stage is the stage of knowledge attainment. During this stage, knowledge of the new guidelines 
and the rationale for change was communicated to all members of the health care team. It is important to stress that these guidelines were developed after many years of research and review. Opportunities for team members to ask questions and express concerns were provided. Once the information and rationale on the new guidelines had been given to the team members, it was important to identify those who were actively seeking more information regarding the implementation and guidelines. The innovators and early adopters were utilized to help incorporate the innovation into clinical practice.

The second stage was the persuasion stage. Employees who showed interest in the guidelines and interventions and were proactive in seeking information and providing solutions were utilized to assist in bringing others on board and spearheading group-wide changes. In addition, during this stage, barriers and problems related to the innovation were identified and addressed. It was beneficial to use the innovators and early adopters to help develop specific processes for implementation of the guidelines.

In the third stage, the decision-making process, the initial decision to incorporate any changes was made. During this process, it was easy to identify those who have not yet adopted the new guidelines or who were resistant to change. If there were a significant number of nonadopters in the group, it might have been prudent to delay the implementation of the guidelines and redirect efforts into the knowledge and persuasion stages. When the decision to implement the new guidelines was made, then formal planning began. In order to plan a significant practice change, all team members needed to fully understand the processes involved. This included development of new tools and restructuring of processes. During this stage, additional barriers and problems were identified and solutions sought. The intermediate stage of initial rejection led to a reevaluation of the innovation (practice change) and the system. If the innovation fails to be 
adopted, then it would be at the initial rejection phase, and the reevaluation will would allow the innovation to be assessed and reintroduced at the beginning, knowledge step, to improve the chance that it would be adopted. The innovators and the early adopters can be utilized for the reevaluation and re-introduction.

Stage four is the implementation phase. During this stage, members of the group worked together to implement the guidelines and processes. The goal was that a majority of the team members would adopt the new guidelines. It has been predicted that if $50 \%$ of the group support an innovation, it has a greater than $80 \%$ chance of successful implementation (Agyeman et al., 2009). Stage five, the confirmation stage, was when the change or guidelines and processes would be formally adopted. At this time, the new guidelines and protocols would be put into routine clinical use. It is important to recognize that even though the intervention or change might now be part of routine practice, it still needs to be evaluated for effectiveness.

While the Diffusion of Innovations theory is a good model for introducing clinical guidelines or practice changes, it does not come without problems. The implementation of a new guideline, process, or practice change is not a fast process, and that needs to be effectively communicated. The initial adoption is a slow process that speeds up as more members of the system agree with the innovation or change. Expectations of a quick adoption process and implementation can lead to problems, disillusion, and ultimately failure to implement the innovation. Interventions that are preventative in nature are harder to adopt because there is no immediate measurable benefit.

The usefulness of this theory has been demonstrated in the corporate, athletic, and practice settings. One of the best resources for the use of this theory is to identify and involve innovators who are excited about practice change, have appropriate levels of knowledge, and are 
considered leaders within the group. One of the barriers to the use of this theory in the clinical practice setting may be a culture of resistance to change. Another barrier could be the general lack of knowledge and understanding regarding the generation of new guidelines and their role in prevention. One of the best ways to assist in implementing any practice change especially in the clinical practice setting is to recognize that change occurs slowly and to begin efforts early in anticipation of a needed practice change. 


\section{Chapter II: Review of the Literature}

\section{Search Strategy}

The articles for review were identified by searching electronic databases. The databases searched were: the Cochrane Library, Academic Search Complete, CINAHL, and Medline. The keywords used were: adherence, guidelines, and lipids. No systematic reviews were identified from the search. The initial search yielded 319 results. The results were narrowed to include only articles published within the last five years, human studies, articles published in peerreviewed journals, and available in English. Seven studies were selected for the review based on the strength of evidence and relevance to the problem. The seven studies included: one randomized controlled trial (Hung, Lin, Hwang, Tsai, \& Li, 2010), and six cross-sectional surveys (Barham et al., 2009; Gowani et al., 2009; Laforest et al., 2008; Lewis, Robinson, Fox, \& Grandy, 2010; Sager et al., 2010; Vulic, Lee, Dede, Lopez, \& Wong, 2010).

\section{Literature Review}

The randomized controlled trial (RCT) evaluated the use of a paper chart reminder on guideline adherence in lipid management in Taiwan (Hung et al., 2010). The study randomized patients with angiographically proven CHD who were not on lipid-lowering therapy (LLT) into either the control group or the intervention group. The intervention was a paper reminder that was placed on the chart that stated "Statins can be beneficial to patients with documented coronary artery disease regardless of their LDL level." Below that statement was the detailed reimbursement policy for the National Health Insurance of Taiwan. If no action was taken after three months, a second reminder was placed on the charts. The study had two end points; the primary endpoint was a new prescription for LLT (statin or ezetimibe) during the six month follow-up period, the secondary endpoint was the composite of LLT or repeat lipid profile within 
six months. The investigators randomized 194 patients, 92 to the study group and 102 to the control group. The groups were well-matched. The inclusion and exclusion criteria were well described. There were new LLT prescriptions in $14.1 \%$ of the study group and $8.8 \%$ of the control group. The odds-ratio (OR) for LLT was 1.70 ( $\mathrm{P}=0.248,95 \%$ CI: $0.69-4.19)$. The secondary endpoint showed better results in the study group. The study group had repeat lipid panels in $59.8 \%$ of the patients. The control group only had $35.3 \%$ of patients with repeat lipid panels. The OR for the secondary endpoint was $2.81(\mathrm{P}=0.001,95 \% \mathrm{CI}: 1.57-5.04)$. The investigators were unable to see a significant improvement in the primary endpoint, but did see an improvement in the secondary endpoint. The inclusion of the reimbursement policy on the reminder is thought to be a significant factor in the lack of improvement in the primary endpoint. The reimbursement policy (which does not reimburse unless LDL $\geq 130$ ) contradicts the other statement that the use of a statin is beneficial regardless of LDL level. The studies strength is its RCT design. Its weaknesses are: the small sample size, lack of ethnic diversity, and policy restrictions that affected the primary endpoint.

There were six cross-sectional surveys (CSS) reviewed. The first CSS evaluated the appropriateness of cholesterol management in primary care. The investigators (Barham et al., 2009) evaluated the appropriateness of cholesterol management in relation to cardiovascular risk and sex. They examined medical records from 60 community practices in North Carolina that were participating in a randomized practice-based trail (Guideline Adherence for Heart Health). They investigated 5031 patients aged 21-84 years. The investigators used a multivariate logistic regression to assess whether age, sex, race, diabetes, cardiovascular disease, ATP-III risk category, or pre-treatment LDL influenced treatment for lipid disorders. They found that screening rates were higher in those who were older and those who had diabetes or 
cardiovascular disease. However, they did not see any large difference in screening rates by sex. They found that older age was associated with less appropriate lipid treatment (OR 0.91, $\mathrm{P}=0.01$ ) even though older adults had higher screening rates. In addition, they found that patients with $\mathrm{LDL} \leq 130$ and those in the low cardiovascular risk category were more likely to be managed appropriately when compared with those whose LDL $\geq 190$ and those at high risk $(\mathrm{OR} 18.8, \mathrm{P}<$ 0.001; OR 27.5, $\mathrm{P}<0.001)$. Investigators also found that of the 375 patients eligible for treatment, those with LDL levels $\geq 131$ and $\leq 159$ were less likely to be treated compared to those with $\mathrm{LDL} \geq 190$ (OR 0.15, $\mathrm{P}=<0.001)$. They concluded that adherence to ATP-III guidelines is more challenging in intermediate-risk and high-risk patients than for those at lowrisk, and patients in those categories were significantly less likely to be managed appropriately. This study's strength is its large sample size and use of multiple practices. It weaknesses are in the CSS design and the data (medical record) abstraction, which may not have captured all of the actual patient data.

Another cross-sectional survey evaluated LDL goal attainment in patients receiving LLT (LaForest et al., 2008). The investigators analyzed 2727 patients obtained from a French computerized general practice database. The patients were selected from randomly chosen providers in the database. They included patients who had a diagnosis of dyslipidemia (hyperlipidemia) and had a visit within the selected time frame. In addition, the patient had to be treated with at least one lipid-lowering agent (LLA). The investigators examined the number of patients who reached therapeutic objective (TO). They found that only $58.5 \%$ of the high-risk patients met the TO for the overall analysis. When the data was analyzed based specifically on cardiovascular risk, the investigators found that significant differences were observed especially at the high risk level. They found that only $42 \%$ of high risk patients met the TO $(\mathrm{P}<0.0001)$. 
In addition, they also found that when patients had a history of CVD and diabetes, there was a higher percentage (57\%) of patients that had reached TO compared to patients with only CVD (42\%), and those who were without CVD but at high risk (32\%) and being treated as primary prevention $(\mathrm{P}<0.0001)$. The strengths of this study are its large sample size and the use of random selection of practices from which to collect data, as well as the large number of practices sampled. Its weaknesses are using guidelines other than ATP-III, although they are similar, and the use of multiple risk categories and sub-categories.

The third cross-sectional survey examined the underutilization of cardiovascular medications in at risk individuals (Lewis, Robinson, Fox, \& Grandy, 2010). The investigators identified patients from the SHEILD (Study to Help Improve Early evaluation and management of risk factors Leading to Diabetes) study who were at risk for or with a history of CVD events such as MI, stroke, or revascularization. CHD risk was defined using the ATP-III guidelines. The respondents were divided into three categories: high CHD risk (7510), moderate CHD risk (4823), and low CHD risk (5307). The investigators found that the use of LLT was very low in each group $(21.5 \%, 13.0 \%, 5.5 \%$ respectively; $\mathrm{p}<0.001)$. After adjusting for age, gender, and geography, they found that high and moderate CHD risk groups were more likely to have gotten LLT than the low-risk participants $(\mathrm{p}<0.001)$. The high-risk group was over four times more likely to have had statin therapy (OR 4.60, 95\% CI: 3.54-5.97) and the moderate-risk group was over three times more likely (OR 3.13, 95\% CI: 2.45-3.99) to have statin therapy than the lowrisk group. In addition, they found that within the high-risk group, participants with a history of prior CVD event got LLT more often (25\%) than those with type II diabetes $(19.5 \% ; \mathrm{p}=0.02)$. The investigators also found that participants who had type II diabetes were receiving LLT more often than those in the moderate and low risk groups $(\mathrm{p}<0.001)$. This study's strength is its 
large sample size and use of established guidelines for quantifying risk (ATP-III). Its weaknesses are its use of self-report data and its examination of only the use of LLT and not the management of HLD.

Another cross-sectional survey assessed adherence to published lipid treatment guidelines among cardiologists in Pakistan (Gowani et al., 2009). The investigators developed a structured questionnaire to collect information regarding: fundamental knowledge of the basic practices in lipid management, evidence of sex or age bias, and demographics including continuing medical education (CME) activities. The questionnaire was piloted with ten cardiologists who were not part of the study. Feedback from the pilot study provided information to the investigators, the questionnaire was shortened, and some of the language was changed to clarify some of the questions. The study participants were 239 cardiologists from multiple centers in Pakistan who completed a questionnaire. The questionnaire had a maximum score of 14 . In addition, data on the cardiologists' education, type of cardiology practiced, and exposure to continuing medical education (CME) was collected. The median score on the questionnaire was nine. The investigators found that there were fewer correct answers from the cardiologists in areas dealing with fundamental issues such as: initial choice of therapy $(77.0 \%)$; the maintenance of therapeutic dose after goal is attained (46.9\%); and lack of aggressive treatment of LDL (16.7\%). In addition, they demonstrated that the cardiologists indicated that there was bias regarding patient age in respect to different LDL goals (41.8\%) and different thresholds for starting therapy (35.1\%). They also identified physician bias towards patients of different sexes. They noted that there were reported differences in thresholds for starting therapy in women (43.5\%) and different LDL targets in women (46.4\%). They found that $73 \%$ of the cardiologists who reported a bias were less aggressive in treating women. In the adjusted analysis, it was noted that years of 
experience, practice in interventional cardiology, and exposure to CME was associated with increased knowledge $(\mathrm{P}=0.005 ; \mathrm{P}=0.041 ; \mathrm{P}=0.001$, respectively). The study's strengths are the use of a pilot study prior to implementation as well as the use of well recognized lipid guidelines (ATP-III). The weaknesses are: absence of reliability and validity information on the questionnaire, lack of ethnic diversity, and a lack of regulation of medical specialty practice in Pakistan however, that was addressed somewhat by the study design.

The fifth cross-sectional survey evaluated the extent of adherence to recommended cardiovascular therapies (Vulic et al., 2010). The investigators used a multi-ethnic sample with CHD. They assessed adherence to recommended therapies for CHD in representative sample of 364 adults with CHD selected from the National Health and Nutrition Examination Survey (NHANES) database. They calculated the proportion of patients who were receiving recommended therapy. The investigators found the treatment rates for LLT were lower in Hispanics $(27 \%)$ and non Hispanic Blacks $(42 \%)$ than in non-Hispanic Whites $(\mathrm{p}<0.01)$. In addition, they also found that lipid-lowering treatment rates were lower in females $(50 \%)$ as compared to males $(67 \%)(\mathrm{p}<0.01)$. The study's strengths are its use of the NHANES database and use of a multi-ethnic sample. Its weaknesses are the small sample size and lack of laboratory data.

The final cross-sectional study reviewed physicians' perception of LDL goals based on guideline recommendations (Sager et al., 2010). The investigators asked 907 physicians to determine the LDL goal for 30 of their patients with HLD based on current guidelines (ATP-III). They divided the 25,250 patients into three different LDL target groups $(<100,<130$, and $<$ 160). The investigators used a logistic regression to evaluate whether the physicians' incorporated risk factors and comorbidities correctly to determine the proper LDL target. They 
found that the physicians identified the correct LDL target in only $55.1 \%$ of male patients versus 49.1\% of females $(\mathrm{P}<0.001)$. The data showed that in the group with LDL target $<100$, the correct assignment was most often assigned to male patients with a history of CAD and myocardial infarction (77.1\%). The investigators found the correct LDL goal of $<100$ in patients with $\mathrm{CAD}$ and concomitant disease. The found the rates to be: $\mathrm{CAD}$ and $\mathrm{CABG}$ (76.6\%), CAD and diabetes (69.6\%), CAD without CABG (61.3\%), CAD without diabetes (61.2 $\%)$, and CAD without MI (58.4\%). They also found in all patients with CAD, that the correct LDL goal was assigned more often in men than in women (67.6\% v. 59.7\%; $<0.001)$. The data was placed in quartiles based on guideline knowledge. They found that the patients of the physicians in the top quartile ( $>90 \%$ correct LDL goals) had lower LDL levels than those in the other quartiles $(\mathrm{p}<0.001)$. The strength of this study is its large sample size. Its only significant weakness is the cross-sectional survey design.

\section{Synthesis}

The RCT (Hung et al., 2010) showed that the use of a paper-based clinical reminder could modify provider behavior in assessing lipid values. Unfortunately, this study did not show an increase in the use of LLT. The inclusion of the reimbursement policy for LLT on the reminder form significantly affected the use of LLT in the participants, which was the primary outcome. The reimbursement policy contradicted the other statement that "statins can be beneficial regardless of LDL level". It is probable that the absence of the reimbursement policy statement would have led to a better outcome in terms of better use of LLT. While the study did not meet its primary outcome, it did adequately demonstrate that the use of a paper-based clinical reminder could modify provider behavior. 
The six cross sectional surveys clearly demonstrated that there is a lack of provider adherence to lipid guidelines. The studies addressed different aspects of adherence to lipidlowering guidelines. Two of the studies (Barham et al., 2009; Laforest et al., 2008) examined lipid management and found that those at high risk were less likely to receive appropriate treatments or reach therapeutic objective (goal). Two of the studies reviewed treatment rates (for LLT) and utilization of LLT (Lewis et al., 2010; Vulic et al., 2010). Those studies showed that the treatment rates were low for all groups studied. Two of the studies (Gowami, et al., 2009; Sager et al., 2010) evaluated provider knowledge and adherence to lipid-lowering guidelines and demonstrated that there was a distinct lack of provider knowledge as well as a failure to identify correct LDL targets. In addition, three of the studies (Gowani et al., 2009; Sager et al., 2010; Vulic et al., 2010) showed that providers were more likely to treat and manage lipids more aggressively in men than in women. Two studies (Barham et al., 2009; Vulic et al., 2010) demonstrated that there was age bias as older age was associated with less appropriate treatment. Also, one study found that there was a lack of adequate treatment in some ethnicities especially Hispanics and non-Hispanic Blacks (Vulic et al., 2010). Four of the studies had large sample sizes (Barham et al., 2009; Laforest et al., 2008; Lewis, et al., 2010; Sager et al., 2010). All four of these studies clearly showed that there is a lack of provider adherence to lipid guidelines. This is especially noted in those patients in the high risk categories in all three of these studies (Barham et al., 2009; Laforest et al., 2008; Lewis, et al., 2010). Other cross-sectional studies (Gowani et al., 2009; Vulic et al., 2010) with smaller sample sizes also showed that there was a lack of provider adherence to lipid-lowering guidelines.

Overall the body of evidence from the cross-sectional surveys clearly demonstrates that not only is there a lack of provider adherence to lipid guidelines, but that those at high risk, some 
minorities, and women are especially vulnerable to undertreatment. There were no significant knowledge gaps identified. The RCT adequately showed that the use of a paper-based clinical reminder modified provider behavior. The hypothesis for the project is that: the use of a simple paper lipid-tracking tool in a primary care practice (without EMR) will improve provider adherence to lipid-lowering guidelines and improve patient care. The objective of the RCT is well matched to the hypothesis of the capstone proposal, that a paper based intervention can modify provider behavior. The body of evidence substantiates the need for protocol change that includes a paper based clinical reminder to help modify provider behavior and improve provider adherence to clinical guidelines (ATP-III) for lipid disorders.

\section{Congruence of Organizations Strategic Plan to the Capstone Project}

The goal of the practice is to provide high quality, efficient, ongoing comprehensive medical care for the patients it serves. A large number of the patients have chronic medical conditions such as diabetes, hypertension, chronic kidney disease, and hyperlipidemia. The primary goal of the project is to improve provider adherence to clinical guidelines for lipid disorders. This is in line with the overall goals of the practice.

\section{Project Objectives}

The hypothesis for the project is that the use of a simple paper lipid-tracking tool in a primary care practice (without EMR) will improve provider adherence to lipid-lowering guidelines and improve patient care. The specific objectives for the project after the protocol change are: (1) to educate the staff regarding lipid guidelines through the use of the lipidtracking form (LTF) and lipid guidelines; (2) to assess the baseline adherence to lipid guideline from chart review using the data collection form (DCF) and data prior to the intervention; (3) to demonstrate an increase in screening for lipid disorders after the intervention; (4) to identify 
from the chart review after the protocol change an improvement in the number of patients with abnormal lipid values who are correctly diagnosed with hyperlipidemia (HLD); (5) to increase in the number of patients that have an identified LDL goal after the protocol change; (6) to increase the number of patients who have the correct LDL goal identified; (7) to identify an improvement in the number of patients who are given the correct medications for HLD; (8) to increase in the number of patients who are getting correct follow-up for lipid disorders. It is anticipated that these changes will be both statistically and clinically significant. 


\section{Chapter III: Methodology}

\section{Project Design}

This capstone project used a descriptive, retrospective, before and after design. The project was designed to evaluate the use of a simple paper tracking form on provider adherence to lipid guidelines (ATP-III). There was a pre and post intervention analysis and specific objective measurements of lipid guideline adherence were compared. There was an analysis of a random sample of patients to determine baseline adherence to lipid guidelines prior to the intervention. These patients were randomly selected from the practice database. During a specific time frame, twenty-three consecutive days, the tracking form was placed on the charts of all patients coming to the office for routine office visits. Patients coming to the office for joint injections or dermatological procedures were excluded. The practice is relatively small, approximately 1500 active patients, with only three providers including the project leader. All patients seen during the evaluation period received the intervention (lipid-tracking form) including those seen by the project leader, but that data was removed prior to analysis in order to eliminate bias. After the intervention period, the lipid tracking forms were collected and analyzed. A random sample of the lipid tracking forms were analyzed for comparison to the baseline data.

\section{Resources}

This project did not require many resources other than the project leader's time. Office supplies such as paper, ink, and photocopying were provided by the site. Educational materials were already present at the site and provided by an outside vendor (non-pharmaceutical). The data collection form and lipid tracking tool were developed and printed by the project leader. There is little or no need for additional involvement of ancillary staff as any work involved in the 
project (making labs slips, scheduling appointments) was already part of their current work duties, and were not in excess. The other providers in the practice were asked to complete the form at the time of the patient visit. This did not significantly impact the time needed for each patient visit. It took less than two minutes to complete the lipid tracking form. Each provider did have a face-to-face in-service on the use of the form as well as written instructions (Appendix A).

The data collection form (DCF) was intended to collect specific objective data on measures of guideline adherence. This form was used to collect the pre and post intervention data. In addition, the form was used to collect baseline demographic data. The data collected included: project identifier (no names were used), date of birth, age, date of last clinic visit, comorbidities (diabetes, coronary artery disease, peripheral vascular disease, stroke), family history of heart disease, date of last lipid profile, diagnosis of HLD, medications for HDL, date of follow-up lipid panel, LDL target, correct LDL target, and whether the patient was at or below LDL target (Appendix B). These data tracking forms were completed by the project leader and were not intended for use by the other providers.

The lipid-tracking form (LTF) was intended it improve provider adherence to lipid guidelines by providing the needed information in one easily accessible place. The form consisted of a grid that provided information on the patients' lipid values, medications, medication changes, planned follow-up, and comments (e.g. missed doses, side effects). The top part of the form included areas for information about the patients' comorbidities (CVD risk), and their LDL goal (Appendix C). In addition, a portion of the ATP-III guidelines (Appendix D) were printed on the form. Also included on the form is the recommended follow-up. This form was intended to be completed by the provider at the time of the patient visit. 


\section{Evidence of Key Site Support}

The site for the implementation of the capstone project was a small independent Internal Medicine practice in Midland County, Michigan. The practice owner was supportive of the project leader's participation in the DNP program. There were multiple discussions between the practice owner and the project leader regarding the project and how it would fit in with the practice. A signed letter allowing the project to be performed at the site was obtained (Appendix E).

\section{Procedure}

The implementation of the practice change occurred in several steps. Prior to implementing the practice change, multiple discussions were held with the other providers in the practice to review the lipid tracking form (LFT) and obtain input on the format. The feedback was utilized in the development of the final form (Appendix C). The providers were given a copy of the ATP-III quick reference guide and the Framingham risk charts (Appendices D \& F). In addition, copies of these were placed in all of the exam rooms. Instructions for the use of the lipid tracking form were given to each provider (Appendix A). Verbal instructions were also provided. The project was submitted the West Virginia University Institutional Review Board (IRB) for review. It was reviewed by the IRB and determined to be exempt.

The evaluation period consisted of 23 consecutive business days. The lipid tracking forms were placed on the charts of the eligible patients by the project leader. This was done just before the patient visit, either the evening before or morning of the visit. The project leader filled out preliminary information on the lipid tracking forms. This included: age, gender, and the most recent lipid values. The providers were expected to fill out the data on comorbidities, determine and document the LDL goal, and indicate medications and follow-up. During the 
evaluation period there were 336 forms placed on the charts. Of the eligible charts, the forms were placed on $100 \%$ of the charts. The forms were left on the charts during the entire evaluation period. After the evaluation period the forms were retrieved. Of the 336 forms that were deployed, 333 were retrieved (99.1\%). Since the forms were placed on the charts just prior to the patient visits, some forms were not eligible to be completed because the patient did not make their appointment. Of the 333 forms that were collected 20 of those where on charts where the patient did not make their visit leaving 313 forms. The sample was then further reduced by excluding the patients that were seen by the project leader yielding a 233 post practice change sample. From this sample 100 forms were randomly selected for analysis. Excluding the project leader only one of the two providers who participated in the evaluation of the practice change completed any of the forms. That provider completed approximately $45 \%$ of the eligible forms.

\section{Evaluation}

The evaluation of the project had several components. The first component was the evaluation of baseline adherence to lipid guidelines. This involved the collection of data on 100 randomly selected patient charts on the data collection form (Appendix B) to track objective markers of adherence (screening, diagnosis, treatment, follow-up, identified LDL goal, and treatment to goal). The second component examined two factors in the implementation of the practice change. The percentage of patients who received the lipid-tracking form was determined. This was accomplished by calculating the number of patients seen during the specified time frame and dividing that into the total number of patients who actually received the lipid-tracking form during that time. This was calculated to be $>99 \%$. In addition, provider compliance with the project was also calculated. This was done by determining the number of patients who received the lipid tracking form and dividing that into the number of completed 
lipid tracking forms. This was also broken down by provider. This was expected to be $>75 \%$. This was to measure implementation of the practice change not patient adherence. The third component was the determination of post-intervention adherence to lipid guidelines. A random sample of the patient's lipid tracking forms were reviewed using the data collection tool and compared to the random patient sample that was analyzed prior to the intervention to determine if the lipid tracking tool improved provider adherence to lipid guidelines. This evaluated specific areas of adherence. In order to compare the results of two groups a chi-square test and independent t-test were used. A p value of $<0.05$ was set for statistical significance. 


\section{Chapter IV: Results}

\section{Sample Characteristics}

There are two samples that will be described in the remainder of this paper: (1) the baseline sample and; (2) the post practice-change sample. The baseline sample (BLS) was a sample consisting of 100 randomly selected charts from the practice cohort. This sample consisted of patients over the age of 20 who did not have the project leader as a primary care provider. The post practice-change sample (PPS) consists of a random selection of 100 patients who received the practice change (LTF). It consisted of patients who were seen by the other providers in the practice. The patients who were seen by the project leader were excluded from selection. The baseline sample $(n=100)$ had a mean age of 67.73 years with a standard deviation of 14.098 years. The minimum age was 37 years and the maximum age was 94 years. The postpractice change sample $(n=100)$ had a mean age of 69.66 years with a standard deviation of 12.173 years. The minimum age was 41 years and the maximum age was 93 years. An independent t-test confirmed that there was no statistically significant difference in the ages of the two samples $(p=0.301)$. The gender distributions in both the pre and post samples were also compared. The baseline sample was $47 \%$ male and 53\% female. In the post practice-change sample was $51 \%$ male and $49 \%$ female. A chi-square test showed there was not a statistically significant difference in gender in the samples $(\mathrm{p}=0.572)$. Race/ethnicity was not analyzed because the baseline characteristic of the practice cohort is predominantly Caucasian (98.66\%). This is consistent with the racial breakdown in Midland County (96.25\%).

\section{Assessment of Baseline Adherence}

To determine baseline adherence to lipid guidelines the baseline sample of patients from the practice cohort were reviewed. Specific criteria were tracked using the data collection form 
(DCF). The criteria were chosen to address the project objectives. The objectives that were addressed in the analysis of baseline adherence included: (1) evaluation of patients who had proper screening; (2) determination of patients who had the correct diagnosis of hyperlipidemia; (3) the number of patients who had a documented LDL goal; (4) the number of patients who had the correct LDL goal; (5) evaluation of the use of correct medication to treat hyperlipidemia; and (6) evaluation of the number of patients who had appropriate follow-up. The assessment was done on data that preceded the practice change. The data collected included: date of visit, date of the most recent labs that preceded the visit, specific comorbidities (diabetes, coronary artery disease, and evidence of need for secondary prevention), lipid values, evidence of LLT, and follow-up.

\section{Screening.}

To determine adherence to screening recommendations, the time between the lab date and the visit date were calculated (lab-days). In addition, follow-up lipid testing was also considered. Based on the calculation of lab-days, it was determined that adequate adherence to lipid screening was achieved at baseline. The mean number of lab-days (number of days between labs and visit) prior to the practice change was 129.77 days, the minimum number of days was 0 and the maximum was 1555 . Recommended screening for patients with chronic disease is annually (364 days) and the recommended screening for those without chronic disease is at least every 5 years (1820 days). An independent t-test showed that there was no association between lab-days and patients who were not at or below goal $(\mathrm{p}=0.533)$.

\section{Assessment of diagnosis of hyperlipidemia.}

The baseline sample was reviewed for patients with abnormal lipid values who did not have evidence of either a diagnosis of hyperlipidemia, (hyperlipidemia, dyslipidemia, or 
hypercholesteremia) or evidence of treatment with lipid lowering therapy. There were not a significant number of patients who had abnormal lipid values who did not have a documented diagnosis or evidence of treatment with lipid lowering therapy. However the absence of documented LDL goals makes this difficult to ascertain. The diagnosis of HLD is relative to the goal. The project leader established estimated goals based on the evidence available for review. Based on the estimated goals there were six patients identified that did not have a diagnosis of HLD (either documented or evidence of LLT) and who were not at or below the goal. A chisquare test showed that this was not statistically significant $(p=0.790)$. At the competition of the project these patient charts were referred to the provider for further review.

\section{Documentation of LDL goal.}

In the baseline sample there we no documented LDL goals in the charts reviewed.

Because there were no documented LDL goals, there was not an opportunity to evaluate whether or not they were the appropriate goals. However, the project leader estimated the LDL goals, based on the information that was available for review in the chart, to determine whether or not the patients were at or below goal. Based on estimated goals determined by the project leader, it was found the $28 \%$ of the sample was not at or below goal, and that $72 \%$ of the patients were at or below the estimated goal.

\section{Assessment of medication usage to treat hyperlipidemia.}

The baseline sample was also analyzed to review the use of medications in the treatment of hyperlipidemia. In addition to the chart review, the electronic prescription system was also reviewed. It was difficult to assess due to individual provider preferences for the use of different medications in the treatment of lipid disorders. In addition, there was lack of adequate documentation regarding the medication choices, especially in those patients not treated with 
statin therapy. While there are appropriate rationales for the use of non-statin therapy, it is useful to have documentation on the reasoning behind the choice of non-statin therapy. The data collection for this project grouped lipid-lowering therapy into four basic categories: 1) statin therapy, 2) Zetia only, 3) over-the-counter (including fish oil, red yeast rice, etc.), and 4) other (Welchol, Lovaza, etc.). In the baseline, sample fifty-seven patients had documented therapy with statins, four were on Zetia alone, five were on over the counter therapies, and five were on other medications. Comparison with the ,at or below goal' (estimated) measure using a chisquare test showed that there were at total of eight patients who were not receiving any treatment who were not at or below goal. This was not statistically significant $(\mathrm{p}=0.814)$.

\section{Follow-up.}

Another indicator of adherence to lipid guidelines that was evaluated is the appropriate follow-up for patients with lipid disorders. This includes visits and labs. The baseline sample was used to determine baseline adherence to follow-up. The determination of follow-up was calculated using chart review and the follow-up guidelines for patients with hyperlipidemia. These follow-up guidelines were also printed on the lipid tracking form. The follow-up guidelines were developed in collaboration with the practice owner. The recommended followup was: (1) new diagnosis, change in medication or unstable, six to twelve weeks; (2) if stable and at goal, three to six months; and (3) patients with chronic disease, annual screening. The project leader reviewed each chart of the patients in the baseline sample. The measure ,followup' used a discrete variable of yes/no. Yes to indicate that there was appropriate follow-up ordered, and no to indicate absence of documented appropriate follow-up. This looked specifically at provider actions (whether or not follow-up was ordered) not at patient compliance 
with the orders. In the baseline sample there were 22 patients who did not have appropriate follow-up and 78 who did.

\section{Evaluation of Post Practice-Change Adherence}

The post practice change adherence to lipid guidelines was evaluated by comparing specific criteria from the baseline sample and the post practice change sample. The specific

criteria compared included: 1) documentation of an LDL goal, and 2) appropriate follow-up for lipids. Age and gender were also compared in the two samples to determine if the samples were equitable. The samples were determined to have similar distributions of age and gender; the details are described further in the sample characteristics section.

\section{Documentation of an LDL goal.}

One of the project objectives was to increase the number of patients who had a documented LDL goal. While the response was not as great as the investigator had originally anticipated, there was an increase in the number of patients who had documented LDL goals, which was statistically significant. Overall there was a $26 \%$ increase in the documentation of an LDL goal. A chi-square test showed that this was statistically significant $(\mathrm{p}=0.000)$. Because only one provider completed the forms, that provider was solely responsible for the $26 \%$ increase. The appropriateness of these documented goals was reviewed based on the information that was readily available in the chart. It was observed that some of the LDL goals were more aggressive than those recommended based on the ATP-III guidelines. This was specifically noted in patients with diabetes. This was not considered an incorrect goal, because other guidelines recommend aggressive lipid lowering in patient with diabetes. Analysis using the more aggressive LDL goals did not indicate and significant difference in the number of patients who were at or below goal. 


\section{Follow-up.}

The post practice change sample was compared to the baseline sample to determine if there was an increase in the number of patients who were given appropriate follow-up for lipid disorders. There was an increase in the number of patients with the proper follow-up ordered after the practice change ( $82 \mathrm{v} .77)$ but it was not statistically significant $(\mathrm{p}=0.457)$. There was a corresponding decrease in the number of patients who did not have follow-up ordered. Twentytwo patients in the baseline sample and eighteen in the post practice change sample.

\section{Other Analyses}

Because only a single provider (other than the project leader) completed the lipid tracking forms during the evaluation period, additional analyses were done using the available data to ascertain if there any other differences between the providers. Lipid data from the combined data set (baseline and post practice change samples) was analyzed with an independent t-test to compare the two providers. There were differences noted between the providers in all lipid values measured (cholesterol, triglycerides, HDL, VLDL, LDL and non-HDL). Provider One who completed the forms during the evaluation period had patients with lower mean levels of cholesterol, triglycerides, VLDL, LDL, and non-HDL than Provider Two. Of those, there were statistically significant differences in cholesterol $(p=0.000)$, LDL $(p=0.009)$, and non-HDL $(p=0.013)$. These are three of the most significant lipid factors in cardiovascular risk.

In addition, the combined data set was used to evaluate any differences in providers for follow-up, ,at or below goal', and use of statin medications. Chi-square analyses were performed. Comparison between Provider One and Provider Two for appropriate follow-up showed that there was a statistically significant difference between the two providers $(p=0.002)$. Provider One had more patients who had appropriate follow-up. Even though Provider Two had 
a higher percentage of patients who were not at or below goal (25.3\% v. $23.1 \%)$ chi-square analysis showed that there was no statistical difference between the two providers $(p=0.727)$. In addition, the two providers were compared on the use of statin medication for the treatment of HLD. There was no difference noted between the two providers in the use of statin medications $(p=0.235)$. 


\section{Chapter V: Summary, Discussion \& Implications}

\section{Congruence with the Theoretical Framework}

This capstone project demonstrated congruence with the theoretical framework. Rogers' Diffusion of Innovation Theory allows for the possibility that the innovation (practice change) might be rejected. Ladd (2010) postulated that there is an additional step of reevaluation that is needed when the innovation is a practice change. This reevaluation step can occur at two different points in the process. After the initial rejection step and after the confirmation step, if the innovation is not formally adopted (Figure 5). This step allows for the members to reassess the practice change and determine if there are any changes that can be made that would make the practice change more compatible with both the members and the practice in general. The general concept for this reevaluation step is that it is difficult to assess whether or not a practice change concept will work until it is introduced into the systems. In addition, if the members know that they will be able to have continuing input into the practice change, they may be more willing to adopt it. The practice change using the lipid tracking form was not formally adopted by the practice. However, the concept was generally well received and while the form in its current state was not adopted, a modified version the form is being developed that will track other chronic diseases as well. Specific aspects regarding the lack of adoption of the practice change were reviewed in the context of Rogers Theory.

\section{Adopter categories.}

The theory identifies five adopter categories: (1) innovators; (2) early adopters; (3) the early majority; (4) the late majority; and (5) laggards. The distribution of the adopter categories usually follows a normal distribution (Figure 6). It can be difficult in a small practice have sufficient numbers for this distribution. Even with intimate knowledge of the system, it is 
difficult to determine into which categories people fall. The innovator for this practice change was the project leader. Provider One was an early adopter, and was involved in the process. Provider Two acted in the manner of a member of the early majority category however, after the evaluation phase, it was discovered that Provider Two was actually a laggard. The system (practice) into which this innovation was introduced did not have the usual distribution in the adopter categories. This is most likely due the small number of providers involved. Other members of the ancillary staff who were in the late majority category did not significantly affect the distribution. In order to assess the congruence of the theory with the practice change, the distribution of the people in each category needs to be assessed at the reevaluation stage to determine if there is sufficient distribution of the adopters in the system for the practice change to be successful in the future.

\section{Factors that affect the rate of adoption.}

In addition, Rogers describes specific factors that affect the rate of adoption. These include: (1) the relative advantage; (2) compatibility within the existing system; (3) complexity; (4) trialability; and (5) observability. In regards to this specific practice change there was a lack of perceived relative advantage. It is difficult to overcome this without insinuating that there is a lack in current practice. The proposed practice change did not possess sufficient compatibility with the current process in the practice. Although it did have compatibility with other practices in the area, with which the staff was familiar. The practice change was not complex and for the evaluation phase of the implementation the majority of the work that would become the responsibility of the ancillary staff was carried out by the project leader. However, were the practice change to have been formally adopted, the ancillary staff would have been required to complete those tasks (inputting the most recent lab values in the form). It may be that they 
perceived that as complex. The reevaluation step (Ladd, 2010) addresses the issue of trialability and was stressed during the process to encourage staff to be more willing to accept the practice change. In addition, the reevaluation step can also demonstrate the relative advantage of the practice change. Because the long term effects of adherence to lipid guidelines are not immediately measurable, there may be a lack of observability. However, there are some aspects of this practice change, such as trends in lipid values, medication changes and adherence to follow-up, that would be documented on the form, that are more observable.

\section{Diffusion of Innovations: Stages.}

This practice change progressed through the stages described by Rogers (1962) and Ladd (2010). It was assumed that the providers (both with many years of experience) had a basic knowledge of the treatment of lipid disorders. That notwithstanding, they were each also provided with copies of the current guidelines. This was the stage of knowledge attainment. The second stage, the persuasion stage, was addressed by involving the providers in the process. Feedback on the lipid-tracking form was obtained and implemented in the final format of the form. In addition, the development of a basic policy for routine follow-up was developed to be incorporated on the form. During this stage, both providers demonstrated involvement in the proposed practice change.

The third stage, the decision making process, involved multiple conversations with the providers and staff regarding how the process would proceed. Specific details such as where the form would be placing in the chart were addressed. Once it was decided that the practice would proceed with the innovation, a timeline was developed. In addition, responsibilities for specific aspects of the practice change were determined. During that stage, it was clear who the innovator, early adopter and early majority participants were. During this stage, it was not 
obvious to the project leader that there were a significant number of non-adopters. Had that been the case the practice change would have needed to be reevaluated.

The fourth stage, the implementation stage, involved the use of the lipid-tracking forms. The implementation began as planned. However, after the first week of the use of the lipidtracking forms, it became apparent that Provider Two was not participating in the practice change at all and that Provider One was not completing as many forms as anticipated. It was unclear to the project leader why this was happening based upon previous indications of support. Therefore, additional conversations occurred with both providers to determine the issue. During these conversations both providers verbalized support for the practice change, and support for the use of the forms. In addition, the instructions for the use of the form (Appendix A) as well as the ATP-III guideline and Framingham risk scores were provided again to the providers. Despite this, Provider Two still did not complete any of the forms. Although Provider One did improve in the number of forms completed, it was still less than what had been anticipated.

The confirmation stage, stage five, is the confirmation stage when the practice is formally adopted. Because of the distinct difference between the providers regarding the use of the form, especially with Provider Two failing to complete any forms, the practice change was not formally adopted. The future of this practice change is technically in the reevaluation stage. However, based on the current practice dynamics, it will not be adopted practice wide. It is suspected by this researcher that one of the biggest obstacles to the implementation of this practice change is the lack of perceived advantage to the current system. It is also suspected that the lack of perceived advantage (over current practice) will be a significant roadblock in the implementation of any new practice change regardless of the system. 
Even with intimate knowledge of the practice setting it is difficult to anticipate how people will behave when faced with change. Even though it was expected that there may have been some resistance to the practice change, and it was believed that those issues had been addressed, the actual outcomes were not anticipated. While the project leader did not expect $100 \%$ participation, it was certainly not anticipated that one of the providers would completely dismiss the practice change and be a laggard. There are several lessons that can be learned from this. The perception of relative advantage on the new practice is crucial to any practice change. The behaviors of participants prior to implementation, while indicative of future behaviors, cannot always be predicted or relied on. The culture of the system (practice) is significant. If the practice has a culture that has been resistant to change, or has not shown evidence of change, the probability that any new practice will be implemented is small, regardless of assurances to the contrary. Words are only as good as the actions that follow.

\section{Discussion}

There is a large body of evidence that has demonstrated that appropriate lipid management reduces cardiovascular morbidity and mortality. In addition, it has been demonstrated that there is a distinct lack of adherence to lipid lowering guidelines for the management of lipid disorders. There is evidence that the use of a paper based intervention could modify behavior. The purpose of this capstone project was to evaluate the use of a paper lipid tracking tool in a primary care setting to determine if it would improve provider adherence to lipid guidelines. One of the key project objectives was to demonstrate that the lipid tracking form increased the number of patient who had documented LDL goals. Determination of a diagnosis of hyperlipidemia is relative to the goal that is determined by the provider. If there is no documented goal or adequate documentation of cardiovascular risk, the patient may be 
undertreated. LDL goals are not static but are changing targets that need to be reevaluated frequently. One of the criticisms of ATP-III is that it is too complicated (Sager, et al., 2010) and leads to too much variation in goals, especially in those at moderate risk.

\section{Limitations of the project.}

This project had several limitations. One limitation was the use of a small practice. The small practice had only two providers (other than the project leader) who were available to utilize the practice change. Since there were only two providers, it became an issue when one provider (Provider Two) failed to participate in the project. This left only one provider to contribute to the evaluation of the practice change. Another limitation of the project was the length of the evaluation period. In order to better evaluate the improvement in the patients' lipid values, a longer study of six months to one year would be needed. In addition, there would need to be better use of the intervention.

\section{Project findings.}

Even with the limitations, there were some positive findings. Analyses of the baseline sample showed that there was already good adherence to some of the measures. There was adherence demonstrated in screening for lipid disorders and documentation of a diagnosis of hyperlipidemia. The evaluation of the use of medications was not able to be done because of individual provider preferences and lack of documentation of rationale. Two measures were compared using the baseline sample and the post practice change samples. These were the documentation of an LDL goal and appropriate follow-up for lipid disorders. This capstone project did demonstrate that there was a statistically significant improvement in the number of patients with documented LDL goals $(p=0.000)$. While there was an increase in the number of patients who had proper follow-up for lipid disorders, it was not statistically significant 
$(\mathrm{p}=0.457)$. The project timeline did not allow for the project leader to assess whether or not there was an increase in the number of patients who were at or below goal.

\section{Implications}

One of the benefits of the lipid tracking form is that it compiles all of the pertinent information in one place. There is no need to search through a chart or remember a rationale. This project did demonstrate that the use of a simple paper lipid tracking from did improve provider adherence to lipid guidelines, but in only one of the four measures. However that measure, documentation of LDL goals, is a critical step in treating hyperlipidemia. It is anticipated that the new guidelines (ATP-IV), due to be released in the fall of 2012, will lower the current goals for primary prevention (Allison, 2010). It will be to provider's and patient's benefit to develop tools to help improve provider adherence. If this simple lipid tracking tool showed improvement even with only one provider participating, it is anticipated that improvement would also be achieved in other practices in which the providers where willing to utilize the forms. In addition, the format could be adapted for use in electronic medical record system so that the information that is important to the management of hyperlipidemia such as, medication, labs, medication changes, and recommended follow-up could be displayed in one screen. This project lays the groundwork for future study. 


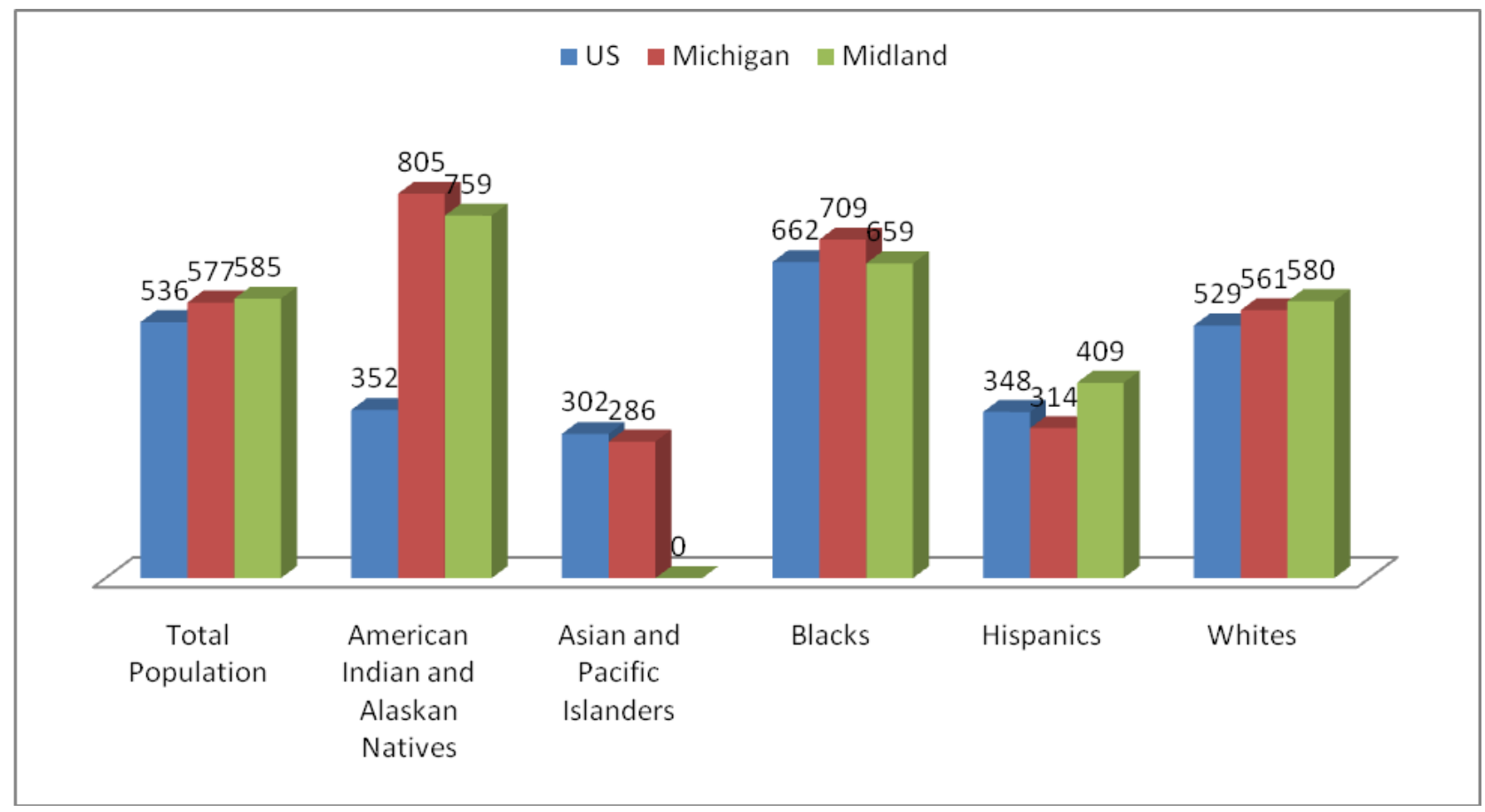

Figure 1: Average annual age-adjusted rate (deaths per 100,000) for people ages 35 years and older (CDC, 2009).

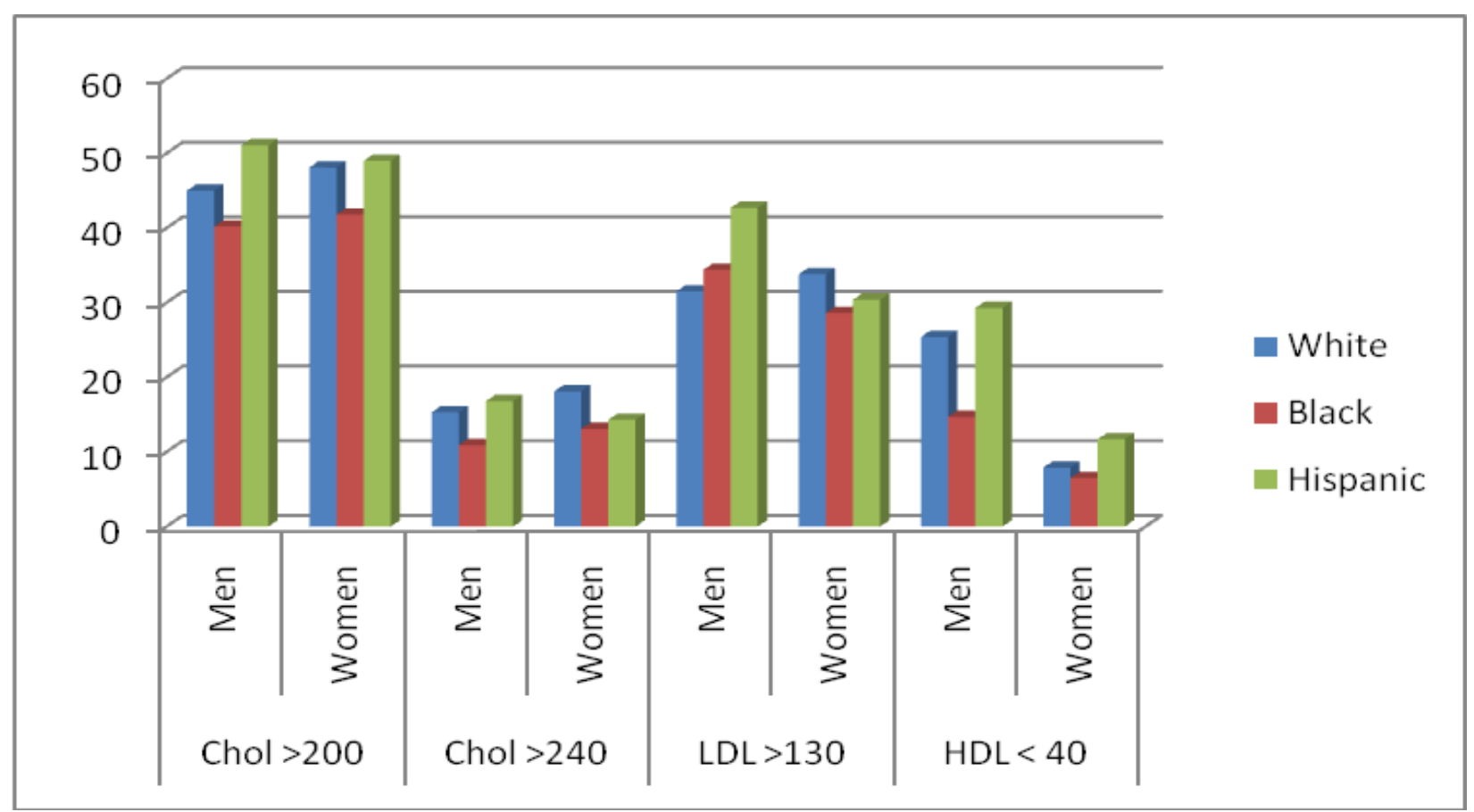

Figure 2: Prevalence of elevated cholesterol and indices of increased cardiovascular risk broken down by gender and race (AHA, 2010). 


\section{Diffusion of Innovations}

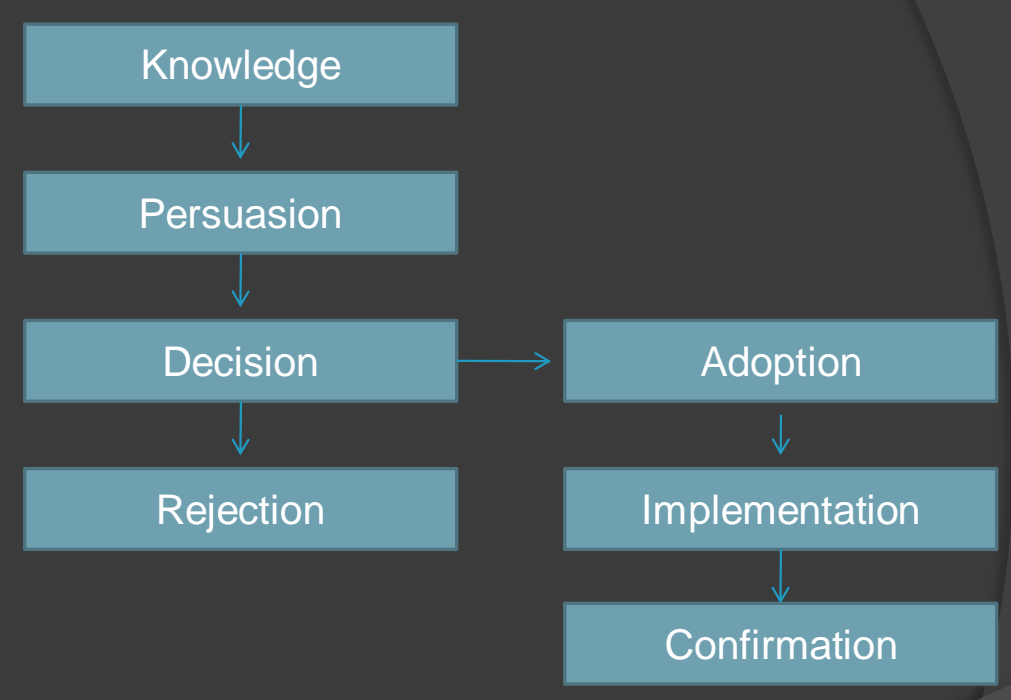

Rogers, 1962

Figure 3: Rogers' Diffusion of Innovation (Rogers, 1962) 


\section{Diffusion of Innovations}

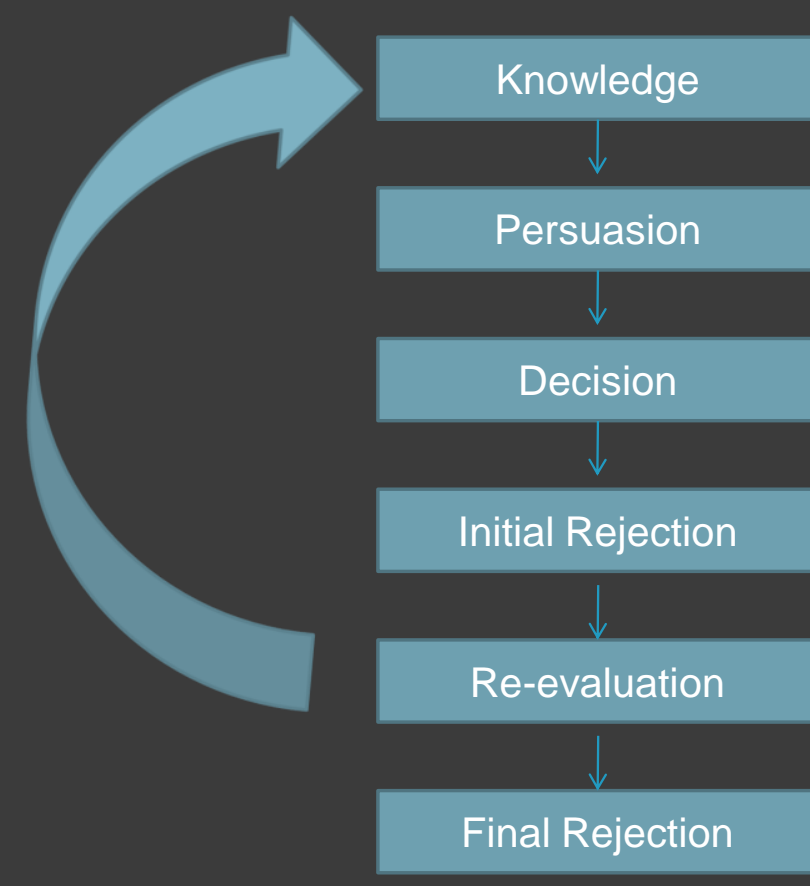

Ladd, 2010; Rogers, 1962

Figure 4: Ladd's (2010) adaption of Rogers' Diffusion of Innovation for use in implementing practice change. 


\section{Diffusion of Innovations}

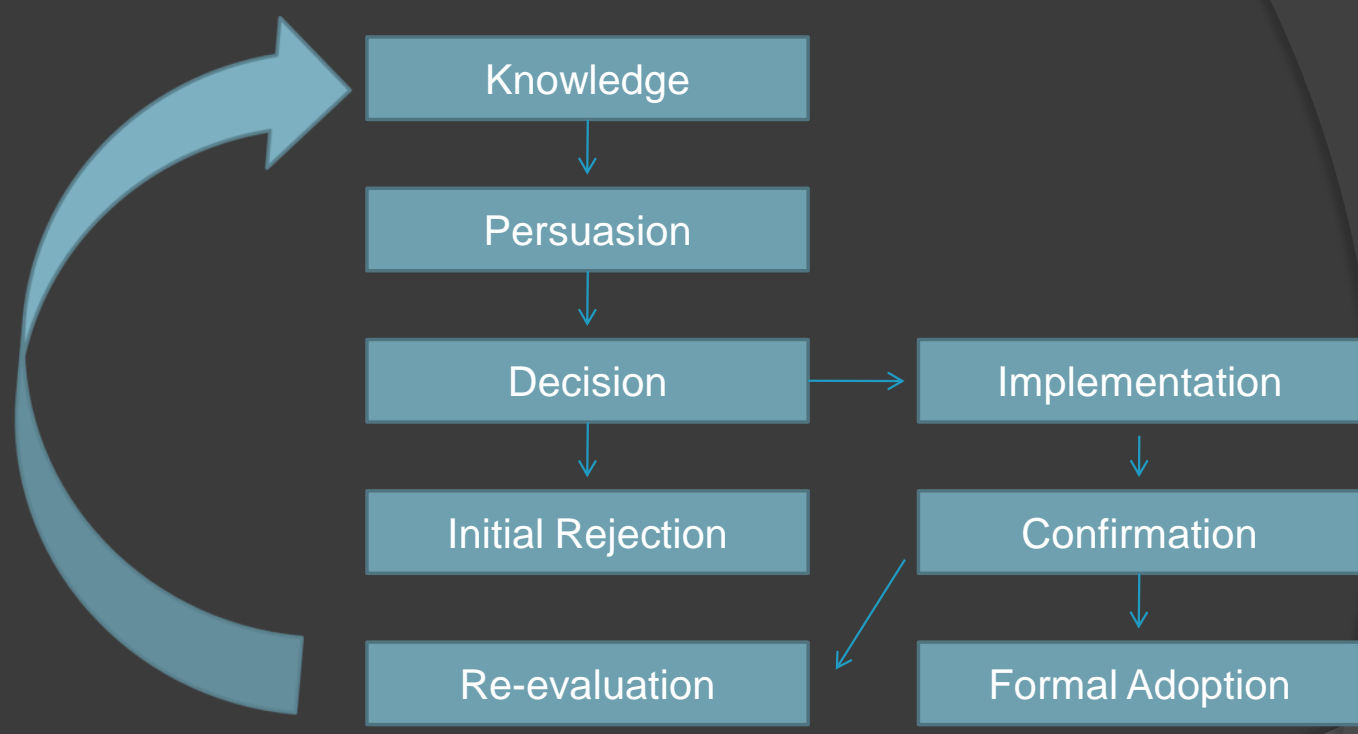

Final Rejection

Ladd, 2010; Rogers, 1962

Figure 5: Ladd's further adaption of Rogers' Diffusion of Innovation for use in implementing practice change. 


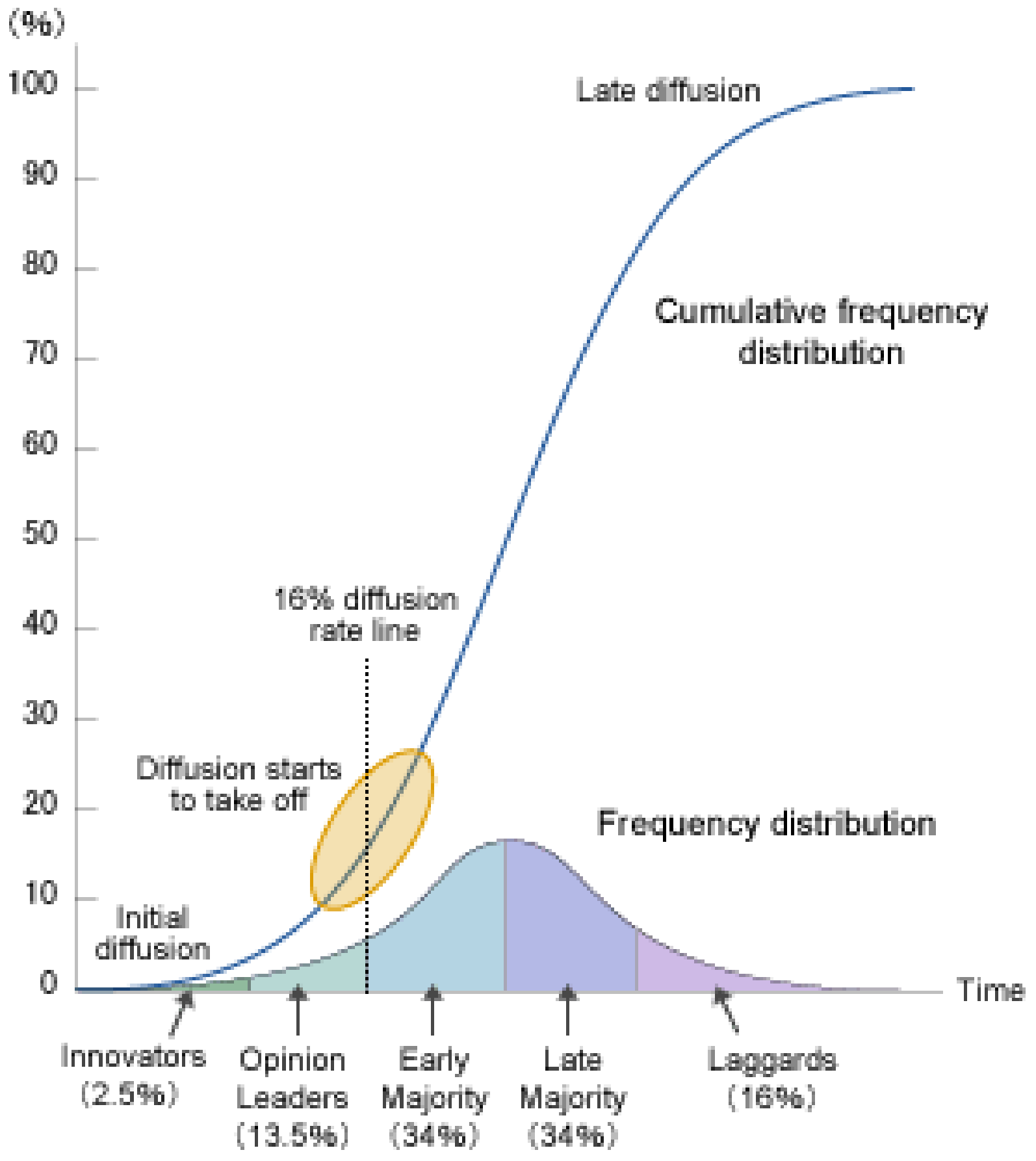

Figure 6: Diffusion of Innovation s-curve and normal distribution of adopter categories (Rogers, 1962) 
Appendix A: Instructions for the Lipid Tracking Form

The form will be placed on the charts of patients (except derm visits and joint injection visits) coming into the office for the next few weeks.

I will be placing the form on the charts after they are prepped and before you see them. I will fill in the basic data (age and gender) and assign a study ID number.

In addition, I will fill in the labs values from the most recent set of labs that are available to me when the chart has been prepped.

When you see the patient, please fill in on the form at the time of the visit the following:

1) Identify and check off the patients comorbidities

2) Determine and document (on the form) the patients LDL goal

3) List the patients current medications (OTC too), indicate none if on no lipid-lowering therapy

4) Note any comments regarding current medication or change in medication (e.g. missed doses, non compliant, increase to Zocor 40, change to Crestor 5 etc.)

5) Document planned follow-up for lipids (e.g. labs 6 weeks, labs 6 months, etc.)

Thanks

Diane 


\section{Appendix B: Lipid Tracking Form}

Ladd 764 LTF

Patient ID:

Age: $\quad$ Gender: $\square \mathrm{M} \quad \square \mathrm{F}$

Comorbidities: $\square$ CAD $\square$ PAD $\square$ AAA $\square$ DM $\quad \square$ Carotid Disease

$\square$ HTN $\square$ Smoking $\square$ Low HDL $\square$ Obesity $\square$ Fam Hx early heart disease $\square$ Unk

Does the patient have a personal history of CABG, Stents, MI, or stroke: $\square$ Yes $\square$ No

If yes; $L D L \leq 70$ for secondary prevention and plaque modification

LDL goal:

\begin{tabular}{|l|l|l|l|l|l|l|l|l|}
\hline Date & & & & & & & \\
\hline Chol & & & & & & & & \\
\hline Trig & & & & & & & & \\
\hline HDL & & & & & & & & \\
\hline VLDL & & & & & & & & \\
\hline LDL & & & & & & & & \\
\hline Non-HDL & & & & & & & & \\
\hline Med & & & & & & & & \\
\hline Med & & & & & & & & \\
\hline Med & & & & & & & & \\
\hline Comment & & & & & & & & \\
\hline Comment & & & & & & & & \\
\hline Comment & & & & & & & & \\
\hline F/U & & & & & & & \\
\hline
\end{tabular}

ATP-III

\begin{tabular}{|l|l|l|}
\hline & LDL Target & $\begin{array}{l}\text { Start Drug } \\
\text { Therapy }\end{array}$ \\
\hline $\begin{array}{l}\text { CHD or CHD } \\
\text { Equivalent } \\
\text { Fram Risk } \geq 20 \%\end{array}$ & $<100 \mathrm{mg} / \mathrm{dL}$ & $\begin{array}{l}\geq 130 \mathrm{mg} / \mathrm{dL} ; \\
\text { If } 100-129 \text { may do } \\
\text { TLC first }\end{array}$ \\
\hline $\begin{array}{l}\geq 2 \text { RF } \\
\text { Fram Risk } 10-20 \%\end{array}$ & $<130 \mathrm{mg} / \mathrm{dL}$ & $\geq 130 \mathrm{mg} / \mathrm{dL}$ \\
Fram Risk $<10 \%$ & & $\geq 160 \mathrm{mg} / \mathrm{dL}$ \\
\hline$<2$ RF & $<160 \mathrm{mg} / \mathrm{dL}$ & $\geq 190 \mathrm{mg} / \mathrm{dL}$ \\
\hline
\end{tabular}

Follow Up for patients on LLT:

6-12 weeks after new diagnosis or change in therapy; visit and/or labs

3-6 months if stable and at goal

Fram Risk = Estimate of 10-year risk for CHD: Framingham point scores

\begin{tabular}{|l|}
\hline \multicolumn{1}{|c|}{ CHD Equiv } \\
\hline PAD \\
\hline AAA \\
\hline DM \\
\hline Carotid dz (Symptomatic) \\
\hline Clinical CHD \\
\hline
\end{tabular}

\begin{tabular}{|l|}
\hline \multicolumn{1}{|c|}{ Risk Factors } \\
\hline HTN \\
\hline Low HDL \\
\hline Family Hx early heart $d z$ \\
\hline Obesity \\
\hline Smoking \\
\hline Age: Males $\geq 45 ;$ Females $\geq 55$ \\
\hline
\end{tabular}




\section{Appendix C: Data Collection Form}

Ladd 764 DCF

Patient ID:

Age:

Date Last Seen:

Comorbidities:

$\square$ PAD

$\square \mathrm{AAA}$

DM

Carotid Disease

$\square$ HTN $\square$ Smoking $\square$ Low HDL $\square$ Obesity $\square$ Fam Hx early heart disease $\square$ Unk Does the patient have a personal history of CABG, Stents, MI, or stroke: $\square$ Yes $\square$ No Date of last lipid panel:

Lipid values: Chol: Trig:

HDL: VLDL: LDL:

Is there a documented diagnosis of hyperlipidemia/dyslipidemia: $\square$ Yes $\square$ No Is there evidence to support a diagnosis of HLD: (or need for LLT eg secondary prevention)

$\square$ Yes $\square$ No Is there evidence of treatment with LLT:

$\square$ Yes $\square$ No

If yes, what:

Does this patient qualify for primary prevention:

$\square$ Yes $\square$ No

Does this patient qualify for secondary prevention:

$\square$ Yes $\square$ No

Is there and identified LDL goal:

$\square$ Yes $\square$ No

If yes, is it correct:

LDL goal:

yes, is it correct:

$\square$ Yes $\square$ No

Correct Goal:

Is the patient at or below goal:

$\square$ Yes $\square$ No

Does the patient have the recommended follow-up (labs and/or visit)

$\begin{array}{lll}\text { 6-12 weeks for new diagnosis or change in therapy } & \square \text { Yes } & \square \text { no } \\ \text { 3-6 months if stable and at goal } & \square \text { Yes } & \square \text { No } \\ \text { If not, documented reason why (out of town, etc) } & \square \text { Yes } & \square \text { No }\end{array}$


Appendix D: ATP-III Quick Reference Guide

National Cholesterol Education Program

\section{ATP III Guidelines At-A-Glance Quick Desk Reference}

Step 1 Determine lipoprotein levels-obtain complete lipoprotein profile after 9- to 12-hour fast.

ATP III Classification of LDL, Total, and HDL Cholesterol (mg/dL)

\begin{tabular}{|c|c|}
\hline \multicolumn{2}{|c|}{ LDL Cholesterol - Primary Target of Therapy } \\
\hline$<100$ & Optimal \\
\hline $100-129$ & Near optimal/above optimal \\
\hline 130-159 & Borderline high \\
\hline 160-189 & High \\
\hline$\geq 190$ & Very high \\
\hline \multicolumn{2}{|c|}{ Total Cholesterol } \\
\hline$<200$ & Desirable \\
\hline $200-239$ & Borderline high \\
\hline$\geq 240$ & High \\
\hline \multicolumn{2}{|c|}{ HDL Cholesterol } \\
\hline$<40$ & Low \\
\hline$\geq 60$ & High \\
\hline
\end{tabular}

Step 2 Identify presence of clinical atherosclerotic disease that confers high risk for coronary heart disease (CHD) events (CHD risk equivalent):

- Clinical CHD

- Symptomatic carotid artery disease

- Peripheral arterial disease

a Abdominal aortic aneurysm.

Step 3

Determine presence of major risk factors (other than $\mathrm{LDL}$ ):

Major Risk Factors (Exclusive of LDL Cholesterol) That Modify LDL Goals

Cigarette smoking

Hypertension ( $\mathrm{BP} \geq 140 / 90 \mathrm{mmHg}$ or on antihypertensive medication)

Low HDL cholesterol $(<40 \mathrm{mg} / \mathrm{dL})^{*}$

Family history of premature $\mathrm{CHD}$ (CHD in male first degree relative $<55$ years;

CHD in female first degree relative $<65$ years)

Age (men $\geq 45$ years; women $\geq 55$ years)

* HDL cholesterol $\geq 60 \mathrm{mg} / \mathrm{dL}$ counts as a "negative" risk factor; its presence removes one risk factor from the total count.

an Note: in ATP III, diabetes is regarded as a CHD risk equivalent. 


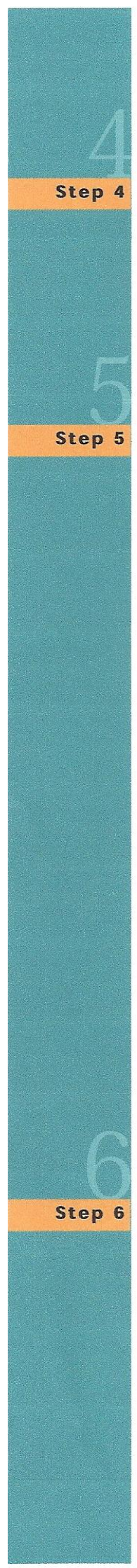

If 2+ risk factors (other than $\mathrm{LDL}$ ) are present without $\mathrm{CHD}$ or $\mathrm{CHD}$ risk equivalent, assess 10-year (short-term) CHD risk (see Framingham tables).

Three levels of 10-year risk:

$$
\begin{aligned}
& =20 \%-\text { CHD risk equivalent } \\
& =10-20 \% \\
& =<10 \%
\end{aligned}
$$

\section{Determine risk category:}

Establish LDL goal of therapy

Determine need for therapeutic lifestyle changes (TLC)

Determine level for drug consideration

\begin{tabular}{|c|c|c|c|}
\hline Risk Category & LDL Goal & $\begin{array}{l}\text { LDL Level at Which } \\
\text { to Initiate Therapeutic } \\
\text { Lifestyle Changes (TLC) }\end{array}$ & $\begin{array}{l}\text { LDL Level at Which to } \\
\text { Consider Drug Therapy }\end{array}$ \\
\hline $\begin{array}{l}\text { CHD or CHD Risk Equivalents } \\
\text { (10-year risk }>20 \%)\end{array}$ & $<100 \mathrm{mg} / \mathrm{dL}$ & $\geq 100 \mathrm{mg} / \mathrm{dL}$ & $\begin{array}{l}\geq 130 \mathrm{mg} / \mathrm{dL} \\
(100-129 \mathrm{mg} / \mathrm{dL}: \text { drug optional })^{*}\end{array}$ \\
\hline \multirow{2}{*}{$\begin{array}{l}2+\text { Risk Factors } \\
\text { (10-year risk } \leq 20 \%)\end{array}$} & \multirow{2}{*}{$<130 \mathrm{mg} / \mathrm{dL}$} & \multirow{2}{*}{$\geq 130 \mathrm{mg} / \mathrm{dL}$} & $\begin{array}{l}\text { 10-year risk } 10-20 \% \text { : } \\
\geq 130 \mathrm{mg} / \mathrm{dL}\end{array}$ \\
\hline & & & $\begin{array}{l}\text { 10-year risk <10\%: } \\
\geq 160 \mathrm{mg} / \mathrm{dL}\end{array}$ \\
\hline 0-1 Risk Factor ${ }^{\dagger}$ & $<160 \mathrm{mg} / \mathrm{dL}$ & $\geq 160 \mathrm{mg} / \mathrm{dL}$ & $\begin{array}{l}\geq 190 \mathrm{mg} / \mathrm{dL} \\
\text { (160-189 mg/dL: LDL-lowering } \\
\text { drug optional) }\end{array}$ \\
\hline
\end{tabular}

LDL Cholesterol Goals and Cutpoints for Therapeutic Lifestyle Changes (TLC) and Drug Therapy in Differen Risk Categories.

* Some authorities recommend use of $L D L$-lowering drugs in this category if an $L D L$ cholesterol $<100 \mathrm{mg} / \mathrm{dL}$ cannot be achieved by therapeutic lifestyle changes. Others prefer use of drugs that primarily modify triglycerides and HDL, e.g., nicotinic acid or fibrate. Clinical judgment also may call for deferring drug therapy in this subcategory.

$\dagger$ Almost all people with 0-1 risk factor have a 10-year risk $<10 \%$, thus 10 -year risk assessment in people with 0-1 risk factor is not necessary.

Initiate therapeutic lifestyle changes (TLC) if LDL is above goal.

TLC Features

TLC Diet:

- Saturated fat $<7 \%$ of calories, cholesterol $<200 \mathrm{mg} /$ day

- Consider increased viscous (soluble) fiber (10-25 g/day) and plant stanols/sterols

(2g/day) as therapeutic options to enhance LDL lowering

- Weight management

increased physical activity. 


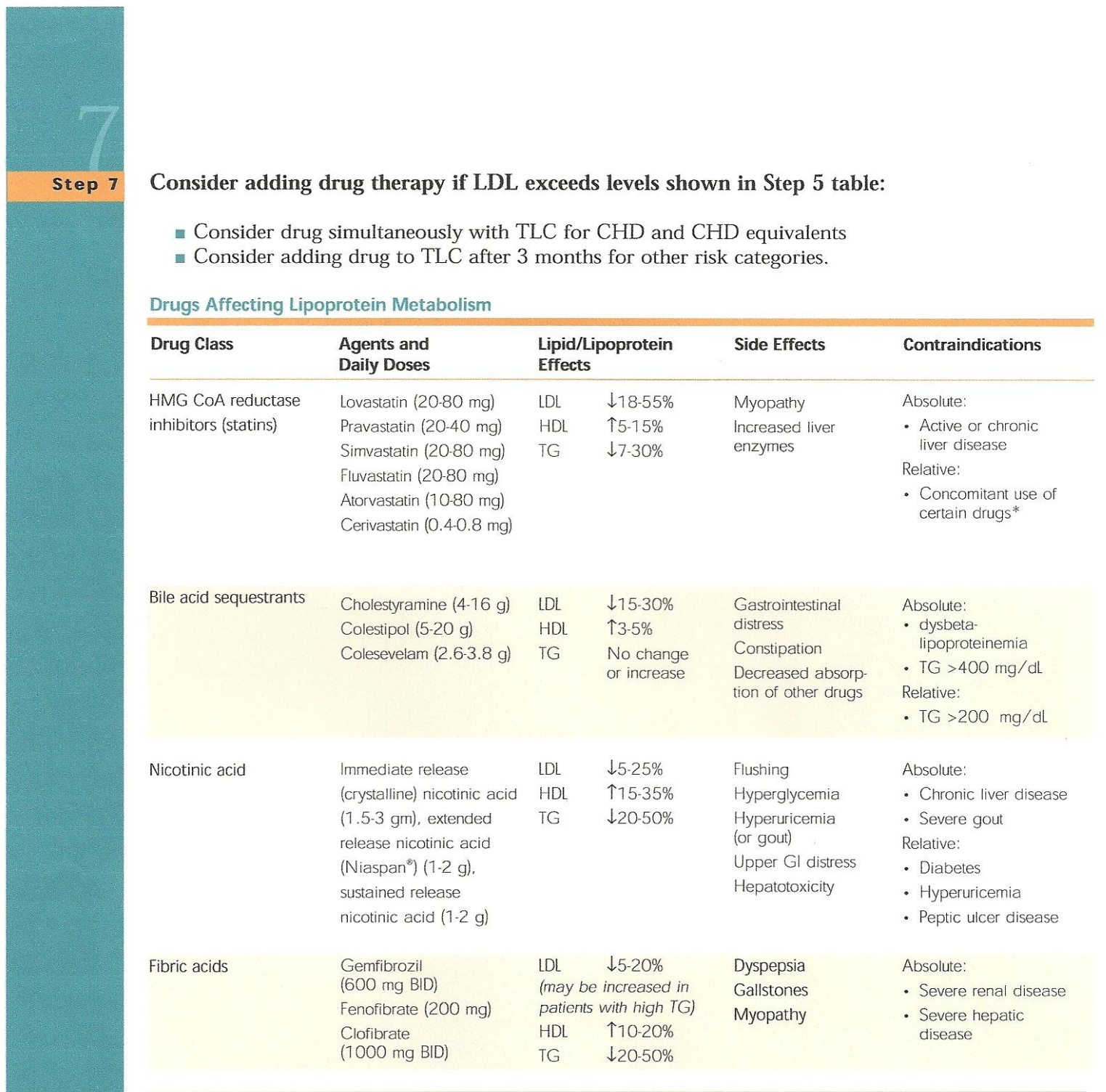

* Cyclosporine, macrolide antibiotics, various anti-fungal agents, and cytochrome P-450 inhibitors (fibrates and niacin should be used with appropriate caution). 


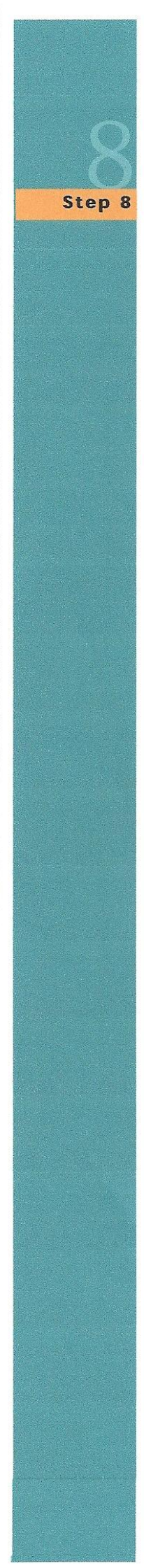

Identify metabolic syndrome and treat, if present, after 3 months of TLC.

Clinical Identification of the Metabolic Syndrome - Any 3 of the Following:

\begin{tabular}{|c|c|}
\hline Risk Factor & Defining Level \\
\hline Abdominal obesity* & Waist circumference ${ }^{\dagger}$ \\
\hline Men & $>102 \mathrm{~cm}(>40 \mathrm{in})$ \\
\hline Women & $>88 \mathrm{~cm}(>35 \mathrm{in})$ \\
\hline Triglycerides & $\geq 150 \mathrm{mg} / \mathrm{dL}$ \\
\hline \multicolumn{2}{|l|}{ HDL cholesterol } \\
\hline Men & $<40 \mathrm{mg} / \mathrm{dL}$ \\
\hline Women & $<50 \mathrm{mg} / \mathrm{dL}$ \\
\hline Blood pressure & $\geq 130 / \geq 85 \mathrm{mmHg}$ \\
\hline Fasting glucose & $\geq 110 \mathrm{mg} / \mathrm{dL}$ \\
\hline \multicolumn{2}{|c|}{$\begin{array}{l}\text { * Overweight and obesity are associated with insulin resistance and the metabolic syndrome. } \\
\text { However, the presence of abdominal obesity is more highly correlated with the metabolic risk } \\
\text { factors than is an elevated body mass index (BMI). Therefore, the simple measure of waist cir- } \\
\text { cumference is recommended to identify the body weight component of the metabolic syndrome } \\
\text { t Some male patients can develop multiple metabolic risk factors when the waist circumference is } \\
\text { only marginally increased, e.g., } 94-102 \mathrm{~cm} \text { (37-39 in). Such patients may have a strong genetic } \\
\text { contribution to insulin resistance. They should benefit from changes in life habits, similarly to } \\
\text { men with categorical increases in waist circumference. }\end{array}$} \\
\hline
\end{tabular}

Treatment of the metabolic syndrome

- Treat underlying causes (overweight/obesity and physical inactivity):

- Intensify weight management

- Increase physical activity.

Treat lipid and non-lipid risk factors if they persist despite these lifestyle therapies:

- Treat hypertension

- Use aspirin for CHD patients to reduce prothrombotic state

- Treat elevated triglycerides and/or low HDL (as shown in Step 9). 


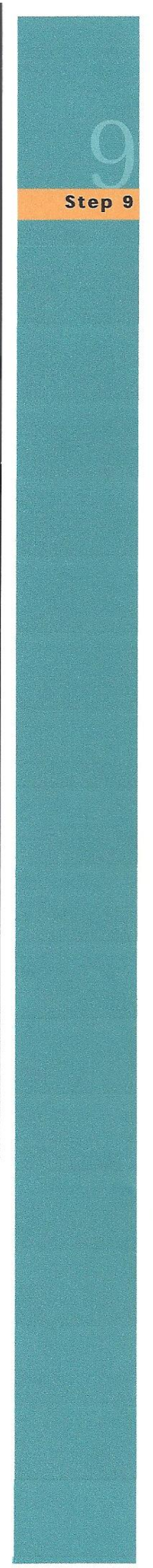

\section{Treat elevated triglycerides.}

ATP III Classification of Serum Triglycerides ( $\mathrm{mg} / \mathrm{dL}$ )

$\begin{array}{ll}<150 & \text { Normal } \\ 150-199 & \text { Borderline high } \\ 200-499 & \text { High } \\ \geq 500 & \text { Very high } \\ & \\ \text { Treatment of elevated triglycerides }(\geq 150 \mathrm{mg} / \mathrm{dL})\end{array}$

- Primary aim of therapy is to reach LDL goal

… Intensify weight management

-1ncrease physical activity

- If triglycerides are $\geq 200 \mathrm{mg} / \mathrm{dL}$ after $\mathrm{LDL}$ goal is reached, set secondary goal for non-HDL cholesterol (total - HDL) $30 \mathrm{mg} / \mathrm{dL}$ higher than LDL goal.

Comparison of LDL Cholesterol and Non-HDL Cholesterol Goals for Three Risk Categories

\begin{tabular}{|c|c|c|}
\hline Risk Category & LDL Goal (mg/dL) & Non-HDL Goal (mg/dL) \\
\hline $\begin{array}{l}\text { CHD and CHD Risk Equivalent } \\
\text { (10-year risk for } \mathrm{CHD}>20 \%)\end{array}$ & $<100$ & $<130$ \\
\hline $\begin{array}{l}\text { Multiple }(2+) \text { Risk Factors and } \\
10 \text {-year risk } \leq 20 \%\end{array}$ & $<130$ & $<160$ \\
\hline 0-1 Risk Factor & $<160$ & $<190$ \\
\hline
\end{tabular}

If triglycerides $200-499 \mathrm{mg} / \mathrm{dL}$ after LDL goal is reached, consider adding drug if needed to reach non-HDL goal:

- intensify therapy with LDL-lowering drug, or

- add nicotinic acid or fibrate to further lower VLDL.

If triglycerides $\geq 500 \mathrm{mg} / \mathrm{dL}$, first lower triglycerides to prevent pancreatitis:

- very low-fat diet $(<15 \%$ of calories from fat)

- weight management and physical activity

- fibrate or nicotinic acid

- when triglycerides $<500 \mathrm{mg} / \mathrm{dL}$, turn to LDL-lowering therapy.

Treatment of low HDL cholesterol $(<40 \mathrm{mg} / \mathrm{dL})$

- First reach LDL goal, then:

- Intensify weight management and increase physical activity

If triglycerides 200-499 mg/dL, achieve non-HDL goal

縉 If triglycerides $<200 \mathrm{mg} / \mathrm{dL}$ (isolated low HDL) in CHD or CHD equivalent consider nicotinic acid or fibrate. 
Appendix E: Letter of Support

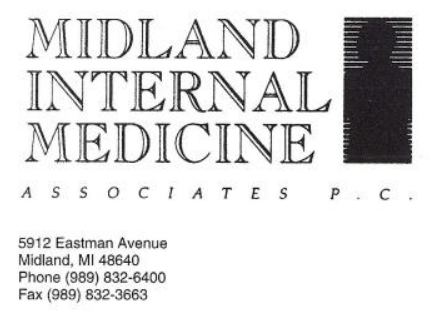

David L. Nadolski, M.D. Sara A. Crawford, ANP-C Diane E. Ladd, FNP - BC Bob Wintermyer, PA-C

To Whom It May Concern:

I have agreed to let Diane Ladd, MSN FNP-BC, DNP student, conduct her Capstone project (The evaluation of the use a lipid tracking form on provider adherence to lipid guidelines) at our practice.
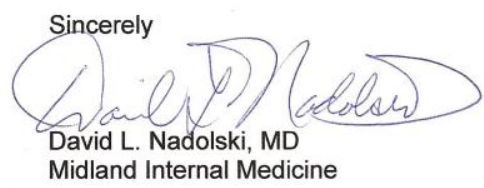

Midland Internal Medicine 


\section{Appendix F: Framingham Risk Scores}

\section{Estimate of 10-Year Risk for Men}

(Framingham Point Scores)

\begin{tabular}{lr} 
Age & Points \\
\hline $20-34$ & -9 \\
$35-39$ & -4 \\
$40-44$ & 0 \\
$45-49$ & 3 \\
$50-54$ & 6 \\
$55-59$ & 8 \\
$60-64$ & 10 \\
$65-69$ & 11 \\
$70-74$ & 12 \\
$75-79$ & 13 \\
\hline
\end{tabular}

\begin{tabular}{lccccc}
\multirow{2}{*}{$\begin{array}{l}\text { Total } \\
\text { Cholesterol }\end{array}$} & \multicolumn{5}{c}{ Points } \\
\cline { 2 - 6 } & Age 20-39 & Age 40-49 & Age 50-59 & Age 60-69 & Age 70-79 \\
\hline$<160$ & 0 & 0 & 0 & 0 & 0 \\
$160-199$ & 4 & 3 & 2 & 1 & 0 \\
$200-239$ & 7 & 5 & 3 & 1 & 0 \\
$240-279$ & 9 & 6 & 4 & 2 & 1 \\
$\geq 280$ & 11 & 8 & 5 & 3 & 1 \\
\hline
\end{tabular}

\begin{tabular}{lccccc} 
& \multicolumn{5}{c}{ Points } \\
\cline { 2 - 6 } & Age 20-39 & Age 40-49 & Age 50-59 & Age 60-69 & Age 70-79 \\
\hline Nonsmoker & 0 & 0 & 0 & 0 & 0 \\
Smoker & 8 & 5 & 3 & 1 & 1 \\
\hline
\end{tabular}

\begin{tabular}{cc} 
HDL $(\mathbf{m g} / \mathbf{d L})$ & Points \\
\hline$\geq 60$ & -1 \\
$50-59$ & 0 \\
$40-49$ & 1 \\
$<40$ & 2 \\
\hline
\end{tabular}

\begin{tabular}{ccc} 
Systolic BP $\mathbf{( m m H g})$ & If Untreated & If Treated \\
\hline$<120$ & 0 & 0 \\
$120-129$ & 0 & 1 \\
$130-139$ & 1 & 2 \\
$140-159$ & 1 & 2 \\
$\geq 160$ & 2 & 3
\end{tabular}

Point Total $\quad$ 10-Year Risk \%

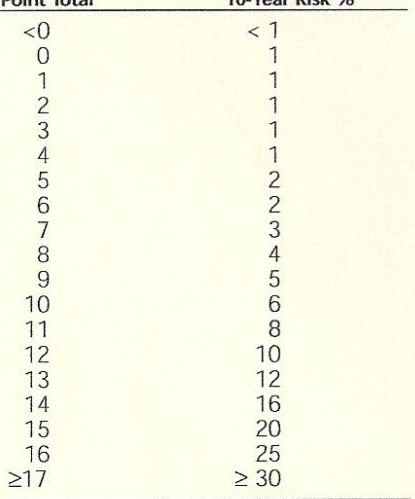

Estimate of 10-Year Risk for Women

(Framingham Point Scores)

\begin{tabular}{lr} 
Age & Points \\
\hline $20-34$ & -7 \\
$35-39$ & -3 \\
$40-44$ & 0 \\
$45-49$ & 3 \\
$50-54$ & 6 \\
$55-59$ & 8 \\
$60-64$ & 10 \\
$65-69$ & 12 \\
$70-74$ & 14 \\
$75-79$ & 16 \\
\hline
\end{tabular}

\begin{tabular}{cccccc}
\multirow{2}{*}{$\begin{array}{l}\text { Total } \\
\text { Cholesterol }\end{array}$} & \multicolumn{5}{c}{ Points } \\
\cline { 2 - 6 } & Age 20-39 & Age 40-49 & Age 50-59 & Age 60-69 & Age 70-79 \\
\hline$<160$ & 0 & 0 & 0 & 0 & 0 \\
$160-199$ & 4 & 3 & 2 & 1 & 1 \\
$200-239$ & 8 & 6 & 4 & 2 & 1 \\
$240-279$ & 11 & 8 & 5 & 3 & 2 \\
$\geq 280$ & 13 & 10 & 7 & 4 & 2 \\
\hline
\end{tabular}

\begin{tabular}{lccccc}
\multicolumn{5}{c}{} & \multicolumn{5}{c}{ Points } \\
\cline { 2 - 6 } \multicolumn{1}{c}{} & Age 20-39 & Age 40-49 & Age 50-59 & Age 60-69 & Age 70-79 \\
\hline Nonsmoker & 0 & 0 & 0 & 0 & 0 \\
Smoker & 9 & 7 & 4 & 2 & 1 \\
\hline
\end{tabular}

\begin{tabular}{cc} 
HDL $(\mathbf{m g} / \mathbf{d L})$ & Points \\
\hline$\geq 60$ & -1 \\
$50-59$ & 0 \\
$40-49$ & 1 \\
$<40$ & 2 \\
\hline
\end{tabular}

\begin{tabular}{ccc} 
Systolic BP $\mathbf{( m m H g})$ & If Untreated & If Treated \\
\hline$<120$ & 0 & 0 \\
$120-129$ & 1 & 3 \\
$130-139$ & 2 & 4 \\
$140-159$ & 3 & 5 \\
$\geq 160$ & 4 & 6
\end{tabular}

$\geq 160$

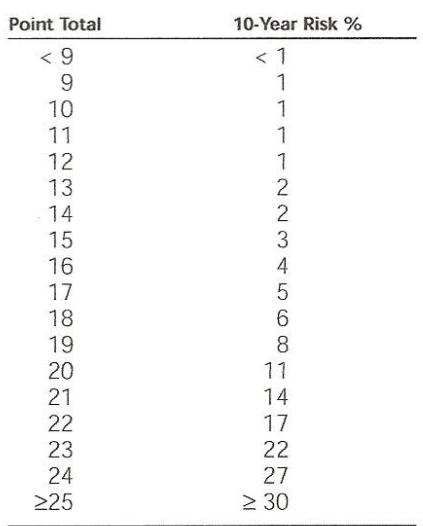

10-Year risk

10-Year risk __ $\%$
U.S. DEPARTMENT OF HEALTH AND HUMAN SERVICES Public Health Servic

National Institutes of Health

National Heart, Lung, and Blood Institute
NIH Publication No. 01-3305

May 2001 


\section{References}

Agyeman, P., Desgrandchamps, D., Vaudaux, B., Berger, C., Diana, A., Heininger, U.,...Aebi, C. (2009). Interpretation of primary care physicians' attitude regarding rotavirus immunisation using diffusion of innovation theories. Vaccine, 27(35), 4771-4775.

Allison, T. G. (2010). Lipid management standard and advanced preview of ATP-IV. Retrieved from: www.cardiologia.spc.org.py/wp-content/.../Lipid-Talk-Paraguay-final.ppt American Heart Association (2010). Heart disease and stroke statistics: 2010 update. http://americanheart.org/presenter.jhtml?identifier $=3000090$

American Heart Association. (2006). Cardiovascular disease cost. http://www.americanheart.org/presenter.jhtml?identifier $=4478$

Barham, A. H., Goff, D. C.,Jr., Chen, H., Balasubramanyam, A., Rosenberger, E., Bonds, D. E., \& Bertoni, A. G. (2009). Appropriateness of cholesterol management in primary care by sex and level of cardiovascular risk. Preventive Cardiology, 12(2), 95-101.

Centers for Disease Control and Prevention. (2009). Leading causes of death. Retrieved http://www.cdc.gov/nchs/FASTATS/lcod.htm

Centers for Disease Control and Prevention. (2009). Leading causes of death by state. http://www.cdc.gov/heartdisease/maps_statistics.htm

Corrao, G., Conti, V., Merlino, L., Catapano, A. L., \& Mancia, G. (2010). Results of a retrospective database analysis of adherence to statin therapy and risk of nonfatal ischemic heart disease in daily clinical practice in italy. Clinical Therapeutics, 32(2), 300-310. doi:10.1016/j.clinthera.2010.02.004 
De Civita, M., \& Dasgupta, K. (2007). Using diffusion of innovations theory to guide diabetes management program development: An illustrative example. Journal of Public Health (Oxford, England), 29(3), 263-268.

Gowani, S. A., Shoukat, S., Taqui, A. M., Bhulani, N., Khalid, S., Sheikh, A.,...Jafary, F. H. (2009). Results of a cross-sectional survey about lipid-management practices among cardiologists in pakistan: Assessment of adherence to published treatment guidelines. Clinical Therapeutics, 31(7), 1604-1614. doi:10.1016/j.clinthera.2009.07.020

Hung, c., Lin, J., Hwang, J., Tsai, R., \& Li, A. (2010). Using paper chart based clinical reminders to improve guideline adherence to lipid management. Journal of Evaluation in Clinical Practice, 14(5), 861-866. doi:10.1111/j.1365-2753.2008.01066.x

Jones, P. H. (2010). Treating dyslipidemia in the high-risk patient. Journal of Family Practice, 59(2), E1-E9.

Karthikeyan, G., Teo, K. K., Islam, S., McQueen, M. J., Pais, P., Wang, X.,...Yusef, S. (2009). Lipid profile, plasma apolipoproteins, and risk of a first myocardial infarction among asians: An analysis from the INTERHEART study. Journal of the American College of Cardiology, 53(3), 244-253.

Ladd, D. E. (2010). Capstone draft proposal: The evaluation of the use of a paper tracking form on improving provider adherence to lipid guidelines. Oral Presentation: West Virginia University. August 2, 2010.

Laforest, L., Moulin, P., Souchet, T., Ritleng, C., Desamericq, G., Le Jeunne, P.,... Van Ganse, E. (2008). Correlates of LDL-cholesterol goal attainment in patients under lipid lowering therapy. Atherosclerosis (00219150), 199(2), 368-377. doi:10.1016/j.atherosclerosis.2007.11.033 
Lewis, S. J., Robinson, J. G., Fox, K. M., \& Grandy, S. (2010). Underutilisation of cardiovascular medications among at-risk individuals. International Journal of Clinical Practice, 64(5), 604-610. doi:10.1111/j.1742-1241.2009.02258.x

Martin, C. S. (2009). The challenge of integrating evidence-based design. Health Environments Research \& Design Journal (HERD), 2(3), 29-50.

McGill, H. C., J., \& McMahan, C. A. (1998). Determinants of atherosclerosis in the young. pathobiological determinants of atherosclerosis in youth (PDAY) research group. The American Journal of Cardiology, 82(10), 30T-36T.

Pasternak, R. C. (2002). 2001 national cholesterol education program (NCEP) guidelines on the detection, evaluation and treatment of elevated cholesterol in adults: Adult treatment panel III (ATP III). ACC Current Journal Review, 11(4), 37-45.

Rogers, E. M. (1962). Diffusion of innovations. Glencoe: Free Press. http://books.google.com/books?id=zw0-AAAAIAAJ

Ryan, B. (1943). The diffusion of hybrid seed corn in two Iowa communities. Rural Sociology, $8(1), 15-24$

Sager, H. B., Linsel-Nitschke, P., Mayer, B., Lieb, W., Franzel, B., Elsasser, U., \& Schunkert, H. (2010). Physicians' perception of guideline-recommended low-density lipoprotein target values: Characteristics of misclassified patients. European Heart Journal, 31(10), 12661273.

Sharma, M., \& Kanekar, A. (2008). Diffusion of innovations theory for alcohol, tobacco, and drugs. Journal of Alcohol \& Drug Education 52(1), 3-7.

Vulic, D., Lee, B. T., Dede, J., Lopez, V. A., \& Wong, N. D. (2010). Extent of control of cardiovascular risk factors and adherence to recommended therapies in US multiethnic adults 
with coronary heart disease: From a 2005-2006 national survey. American Journal of Cardiovascular Drugs: Drugs, Devices, and Other Interventions, 10(2), 109-114. doi: $10.2165 / 11535240-000000000-00000$

Yusuf, S., Hawken, S., Ôunpuu, S., Dans, T., Avezum, A., Lanas, F.,...Lisheng, L. (2004). Effect of potentially modifiable risk factors associated with myocardial infarction in 52 countries (the INTERHEART study): Case-control study. Lancet, 364(9438), 937-952.

\section{John H. ou=Acquisitions Department,

